\title{
Ultimate limits of approximate unambiguous discrimination
}

\author{
Quntao Zhuang ๑* \\ Department of Electrical and Computer Engineering, University of Arizona, Tucson, Arizona 85721, USA \\ and James C. Wyant College of Optical Sciences, University of Arizona, Tucson, Arizona 85721, USA
}

(Received 29 July 2020; accepted 3 November 2020; published 23 November 2020)

\begin{abstract}
Quantum hypothesis testing is an important tool for quantum information processing. Two main strategies have been widely adopted: in a minimum error discrimination strategy, the average error probability is minimized; while in an unambiguous discrimination strategy, an inconclusive decision (abstention) is allowed to vanish any possibility of errors when a conclusive result is obtained. In both scenarios, the testing between quantum states is relatively well understood, for example, the ultimate limits of the performance are established decades ago; however, the testing between quantum channels is less understood. Although the ultimate limit of minimum error discrimination between channels has been explored recently, the corresponding limit of unambiguous discrimination is unknown. In this paper, we formulate an approximate unambiguous discrimination scenario, and derive the ultimate limits of the performance for both states and channels. In particular, in the channel case, our lower bound of the inconclusive probability holds for arbitrary adaptive sensing protocols. For the special class of "teleportation-covariant" channels, the lower bound is achievable with maximum entangled inputs and no adaptive strategy is necessary.
\end{abstract}

DOI: 10.1103/PhysRevResearch.2.043276

\section{INTRODUCTION}

Quantum sensing [1-4] has enabled quantum advantages in various applications, such as positioning and timing [5,6], target detection [7-11], digital-memory reading [12], distributed sensing [13-18], entangled-assisted spectroscopy [19], and most prominently the Laser Interferometer Gravitationalwave Observatory (LIGO) [20-22]. As fundamental tools for quantum sensing, various different strategies have been developed for quantum hypothesis testing in various scenarios. In a minimum error discrimination (MED) [23-26] strategy, the overall error probability is minimized, while in an unambiguous discrimination (UD) [27-31] strategy, an inconclusive result is allowed to vanish any possible errors. Both strategies have wide applications: MED is important for applications like target detection and digital-memory reading; and UD can be utilized in applications related to quantum key distribution protocols [32] and optimal cloning [33]. To make UD practically relevant, given that the experimentation of sensing protocols is never perfect, relaxations of exact UD have been considered in many different approaches, including allowing a fixed inconclusive probability [34-40], maximum confidence [41,42], error-margin tuning [43], and general cost-function approaches $[40,44,45]$. Extensions to parameter-estimation scenarios have led to quantum metrology protocols assisted by abstention [46-48].

\footnotetext{
*zhuangquntao@email.arizona.edu

Published by the American Physical Society under the terms of the Creative Commons Attribution 4.0 International license. Further distribution of this work must maintain attribution to the author(s) and the published article's title, journal citation, and DOI.
}

Another complication beyond the different strategies is that the hypotheses being discriminated often involve physical processes modeled as quantum channels. While quantum state discrimination $[24,49,50]$ is relatively well understood, many open problems in quantum channel discrimination [51-55] still await answers. For example, the ultimate limit of state MED [23], the general condition of UD [31,54,56], and the optimum UD of various ensembles of states [57-62] are known. However, quantum channel discrimination is complicated by the various choices of input states and the potential of an adaptive strategy. Due to the complication, the ultimate limits of channel discrimination are much more challenging to solve. While the Helstrom limit [23] is established half a century ago, the ultimate limit of MED between quantum channels has been unsolved until very recently $[63,64]$. And the ultimate limit of UD between quantum channels, beyond the exact UD condition [54], is not well understood.

In this paper, we solve the ultimate limit of an approximate version of UD between quantum channels. Different from previous approaches, we enable the fine tuning of all conclusive conditional error probabilities. Such a relaxation allows the proof of a general continuity inequality for states. Then, we prove an ultimate lower bound on the inconclusive probability in approximate channel UD for an arbitrary adaptive sensing protocol, following Refs. $[63,64]$. The lower bound can be calculated from the approximate UD between Choi states. For a special ensemble of channels called "jointly teleportationcovariant" channels, this lower bound can be achieved by inputting the maximum entangled states and directly measuring the output, without any adaptive strategy involved. When the Choi states are pure, this achievable lower bound can be directly calculated; while for mixed Choi states, we further obtain an efficiently calculable but nontight lower bound. 


\section{APPROXIMATE UNAMBIGUOUS DISCRIMINATION BETWEEN STATES}

Consider an ensemble of states $\boldsymbol{\Upsilon}=\{\boldsymbol{\rho}, \boldsymbol{P}\}$, where the states $\boldsymbol{\rho}=\left\{\rho_{n}\right\}_{n=1}^{m}$ have prior probabilities $\boldsymbol{P}=\left\{P_{n}\right\}_{n=1}^{m}$. The goal of a state discrimination task is to determine an unknown state sampled from the ensemble through a measurement. In general, we also allow an inconclusive decision, when not enough information is obtained to reach a definitive conclusion. Therefore, the measurement is described by a set of positive-operator-valued measurements (POVMs) $\Pi=$ $\left\{\Pi_{n}\right\}_{n=0}^{m}$, where $\Pi_{0}$ represents the inconclusive result, and each of the rest $\Pi_{n}$ corresponds to the decision that the state is $\rho_{n}$. As POVMs, we require each element $\Pi_{n} \geqslant 0$ to be positive semidefinite, and $\sum_{n=0}^{m} \Pi_{n}=I$, with $I$ being the identity operator.

The performance of the protocol is characterized by an overall inconclusive probability

$$
P_{F}=\sum_{n} P_{n} \operatorname{tr}\left(\rho_{n} \Pi_{0}\right),
$$

where $F$ stands for failure, and the conditional conclusive error probabilities

$$
P_{E \mid n}=1-\left[\operatorname{tr}\left(\rho_{n} \Pi_{n}\right)+\operatorname{tr}\left(\rho_{n} \Pi_{0}\right)\right],
$$

where $E$ stands for error.

In the exact UD scenario, one requires all $P_{E \mid n}$ to be zero; in this paper, we introduce the approximate version of UD, where each of the conclusive error probabilities $P_{E \mid n}$ is bounded, forming the set of constraints

$$
\mathbb{E}_{U}(\boldsymbol{\epsilon})=\left\{P_{E \mid n} \leqslant \epsilon_{n}\right\}_{n=1}^{m},
$$

where $\boldsymbol{\epsilon}=\left\{\epsilon_{n}\right\}_{n=1}^{m}$ describes the error tolerance. Alternatively, we can also consider the error probability conditioned on making a conclusive decision. Namely, the constraints become

$$
\mathbb{E}_{R}(\boldsymbol{\epsilon})=\left\{\frac{P_{E \mid n}}{1-P_{F}} \leqslant \epsilon_{n}\right\}_{n=1}^{m} .
$$

Under the above constraint, in general we want to choose the POVM $\Pi$ to minimize the inconclusive probability to obtain

$$
P_{F}^{X \star}(\boldsymbol{\epsilon} ; \Upsilon)=\min _{\Pi: \mathbb{E}_{X}(\boldsymbol{\epsilon})} P_{F}(\Upsilon, \Pi)
$$

as a function of the state ensemble $\Upsilon$ and the constants $\boldsymbol{\epsilon}$. Here, $X$ is a superscript to denote the different constraints: when $X=U$ we adopt the unrescaled constraints in Eq. (3); when $X=R$ we adopt the rescaled constraints in Eq. (4).

The different constraints can be adopted for different purposes. The constraints in Eq. (4) vary quickly when $P_{F}$ is close to unity, therefore leading to complexity in terms of obtaining a good continuity bound. Moreover, as we will show in Lemma $1, P_{F}^{U \star}$ is convex in the constraints $\epsilon$, while $P_{F}^{R \star}$ is not. However, $P_{F}^{R \star}$ is often easier to evaluate.

As the constraints are closely connected by a rescaling, we expect a close connection between $P_{F}^{U \star}$ and $P_{F}^{R \star}$. Reference [37] minimizes the overall error probability given a fixed $P_{F}$, and two constraints are indeed equivalent there. In our case, the formulation is slightly more involved. We can show that when $P_{F}^{U \star}(\boldsymbol{\epsilon} ; \Upsilon)$ as a function of $\boldsymbol{\epsilon}$ is strictly convex or
$P_{F}^{U \star}(\mathbf{0} ; \mathbf{\Upsilon})<1$, then we have (proof in Appendix B)

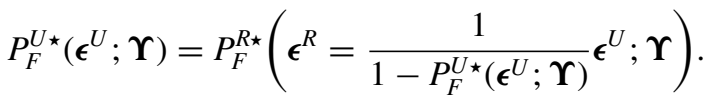

Here we utilize superscripts $U$ or $R$ in the dummy variables $\epsilon$ in the constraints for clarity.

Our formulation allows an interpolation between UD and MED. The case of $\boldsymbol{\epsilon}=\mathbf{0}$ in both formulations corresponds to the (exact) UD scenario. When exact UD is possible, we have $P_{F}^{X \star}(\mathbf{0} ; \mathbf{\Upsilon})<1$; while if exact UD is not possible, we have $P_{F}^{X \star}(\mathbf{0} ; \Upsilon)=1$, which can be achieved by answering "inconclusive" all the time $\left(\Pi_{0}=I\right)$. By fixing the inconclusive probability to be zero, one can obtain the Helstrom limit

$$
P_{H}(\Upsilon)=\min _{\boldsymbol{\epsilon}: P_{F}^{X \star}(\boldsymbol{\epsilon} ; \Upsilon)=0} \boldsymbol{P} \cdot \boldsymbol{\epsilon}
$$

and therefore recover the MED case.

To illustrate the scenario, we consider a pair of nonidentical pure states $\{|p\rangle,|q\rangle\}$, with a general prior $\{p, q\}$ and overlap $\langle p \mid q\rangle=\xi>0$. We denote this ensemble $\Upsilon_{p, q}$ for simplicity. Following the ancilla-assisted measurement protocol $[28,29,65]$, we can solve the rescaled problem with constraint $\left\{\epsilon_{p}, \epsilon_{q}\right\}$ and obtain (see Appendix A for details) the inconclusive probability

$$
\begin{aligned}
& P_{F}^{R \star}=g\left(\xi ; \epsilon_{p}, \epsilon_{q}\right) \equiv \min _{\tilde{\delta}, \tilde{\beta}} p \sin ^{2} \tilde{\beta}+q \sin ^{2} \tilde{\delta}, \\
& \tilde{\delta}, \tilde{\beta}:(\xi-\sin \tilde{\beta} \sin \tilde{\delta}) /(\cos \tilde{\beta} \cos \tilde{\delta}) \in\left[\epsilon_{p q-}, \epsilon_{p q+}\right],
\end{aligned}
$$

where $\epsilon_{p q \pm}=\left|\sqrt{\epsilon_{p}\left(1-\epsilon_{q}\right)} \pm \sqrt{\epsilon_{q}\left(1-\epsilon_{p}\right)}\right|$. One can also obtain a lower bound

$$
P_{F}^{R \star} \geqslant h\left(\xi ; \epsilon_{p}, \epsilon_{q}\right) \equiv 2 \sqrt{p q}\left(1-\frac{1-\xi}{1-\epsilon_{p q+}}\right) .
$$

This lower bound is achievable when $p=q=\frac{1}{2}$, from which we can see that convexity does not hold for the rescaled inconclusive probability.

Because the ensemble of two nonidentical pure states allows exact UD, we have $P_{F}^{X \star}\left(\mathbf{0} ; \Upsilon_{p, q}\right)<1$ and therefore Eq. (6) holds true and we can obtain the unrescaled inconclusive probability from

$$
P_{F}^{U \star}\left[\left\{\epsilon_{p}^{U}, \epsilon_{q}^{U}\right\} ; \Upsilon_{p, q}\right]=g\left(\xi ; \epsilon_{p}, \epsilon_{q}\right),
$$

where the constants $\epsilon_{p, q}^{U}=\left[1-g\left(\xi ; \epsilon_{p}, \epsilon_{q}\right)\right] \epsilon_{p, q}$. One can consider $\left\{\epsilon_{p}, \epsilon_{q}\right\}$ as the parametrization and obtain the overall curve of $P_{F}^{U \star}$.

In Fig. 1, we plot the contour of the minimum inconclusive probability $P_{F}^{R \star}$ with $\epsilon_{p}, \epsilon_{q}$. In Fig. 1(a), we utilize Eq. (9) for the equal prior case. In Fig. 1(b), we numerically solve Eq. (8) for a asymmetric case of $p=\frac{1}{3}$ and $q=\frac{2}{3}$. Points with an equal error probability, $\boldsymbol{P} \cdot \boldsymbol{\epsilon}=$ const, lie on a surface orthogonal to the vector $\boldsymbol{P}$ (blue dashed line). Since we require $P_{F}=0$, the minimum $\boldsymbol{P} \cdot \boldsymbol{\epsilon}$ is achieved when the equal error probability line is tangent to the region of $P_{F}=0$, as shown by the orange dashed line. The optimum choice of $\epsilon$ lies at the tangent point (red cross).

Now, we derive properties of the constrained minimum inconclusive probability. 

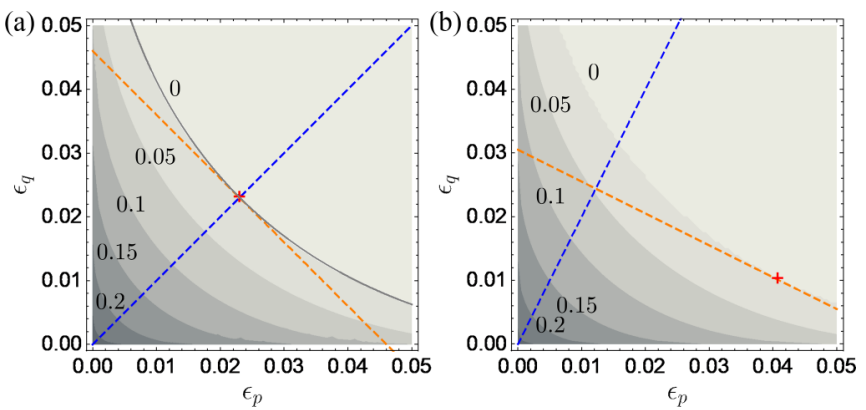

FIG. 1. (a) Contour of the symmetric case, Eq. (9), of prior $p=q=\frac{1}{2}$ and overlap $\xi=0.3$. The red cross indicates the Helstrom limit is achieved. The blue dashed line is $\epsilon_{q}=\epsilon_{p}$. (b) Contour of the asymmetric case, Eq. (8), of prior $p=\frac{1}{3}, q=\frac{2}{3}$, and overlap $\xi=0.3$. The red cross indicates the Helstrom limit is achieved. The blue dashed line represents the vector $\boldsymbol{P}=\left(\frac{1}{3}, \frac{2}{3}\right)$. The orange dashed line is a tangent line to the $P_{F}^{U \star}=0$ boundary.

\section{A. Convexity}

First, we show the convexity of the inconclusive probability as a function of the error constraints.

Lemma 1. Convexity: Consider constants $\left\{\boldsymbol{\epsilon}^{(k)}\right\}_{k=1}^{K}$ and non-negative numbers $\left\{r_{k}\right\}_{k=1}^{K}$ such that $\sum_{k=1}^{K} r_{k}=1$, for any ensemble of states $\boldsymbol{\Upsilon}=\{\boldsymbol{\rho}, \boldsymbol{P}\}$ we have

$$
P_{F}^{U \star}\left(\sum_{k=1}^{K} r_{k} \boldsymbol{\epsilon}^{(k)} ; \boldsymbol{\Upsilon}\right) \leqslant \sum_{k=1}^{K} r_{k} P_{F}^{U \star}\left(\boldsymbol{\epsilon}^{(k)} ; \Upsilon \mathbf{\Upsilon}\right)
$$

The proof of this lemma is via constructing mixed strategies to achieve the performance on the right side of Eq. (11) (see Appendix $\mathrm{C}$ for a detailed proof). Note that for the definition $P_{F}^{R \star}$ in Eq. (5), convexity is not true, due to the extra factor in the constraint in Eq. (4).

Convexity can be useful for obtaining bounds on the performance by extending some operation points. We have the

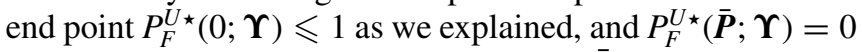
from the random guessing strategy. Here $\overline{\boldsymbol{P}}=\left\{1-P_{k}\right\}_{k=1}^{m}$ is the complement of the prior. Applying Eq. (11) for $K=2$, we have an upper bound on the binary state optimum inconclusive probability. For example, for a symmetric prior, we have $P_{F}^{U \star}(\{\epsilon\} ; \Upsilon) \leqslant 1-\epsilon m /(m-1)$. Better bounds can be achieved by considering the convex hull of $P_{F}^{U \star}(0 ; \Upsilon) \leqslant 1$ and the point achieving the Helstrom limit.

\section{B. A data-processing inequality and lower bounds}

Similar to other sensing scenarios, we can obtain a dataprocessing inequality. Formally, for any quantum channel $\Lambda$, we have

$$
P_{F}^{X \star}\left(\boldsymbol{\epsilon}^{X} ;\{\Lambda(\rho), \boldsymbol{P}\}\right) \geqslant P_{F}^{X \star}\left(\boldsymbol{\epsilon}^{X} ;\{\boldsymbol{\rho}, \boldsymbol{P}\}\right),
$$

for both $X=U, R$, as in a measurement, channel $\Lambda$ can always be applied on states first if one chooses to.

Using this data-processing inequality, we can obtain several lower bounds on the inconclusive probability. Consider mixed states $\rho$ with purifications $\psi_{\rho}=\left\{\psi_{\rho_{n}}\right\}_{n=1}^{m}$ because $\rho$ can be obtained by getting rid of the ancilla that purifies each state, Eq. (12) leads to

$$
P_{F}^{X \star}\left(\boldsymbol{\epsilon}^{X} ;\{\boldsymbol{\rho}, \boldsymbol{P}\}\right) \geqslant P_{F}^{X \star}\left(\boldsymbol{\epsilon}^{X} ;\left\{\boldsymbol{\psi}_{\rho}, \boldsymbol{P}\right\}\right),
$$

for both $X=U, R$. Utilizing Uhlmann's theorem that the maximum overlap between purification equals the fidelity $\left|\left\langle\psi_{\rho_{p}} \mid \psi_{\rho_{q}}\right\rangle\right| \leqslant F\left(\rho_{p}, \rho_{q}\right) \equiv \operatorname{tr}\left(\sqrt{\sqrt{\rho_{q}} \rho_{p} \sqrt{\rho_{q}}}\right)$, we have the following lemma (see Appendix $\mathrm{E}$ for a proof).

Lemma 2. Consider a pair of general nonidentical quantum states $\left\{\rho_{p}, \rho_{q}\right\}$ with prior $\{p, q\}$, the minimum failure probability as a function of the rescaled tolerance $\left\{\epsilon_{p}^{R}, \epsilon_{q}^{R}\right\}$ satisfies

$$
P_{F}^{R \star}\left(\left\{\epsilon_{p}^{R}, \epsilon_{q}^{R}\right\} ; \Upsilon\right) \geqslant P_{F, L B, 1}^{R \star} \equiv g\left(F\left(\rho_{p}, \rho_{q}\right) ; \epsilon_{p}^{R}, \epsilon_{q}^{R}\right) .
$$

Equality is achieved when both states $\rho_{p}, \rho_{q}$ are pure.

A further analytical lower bound can be obtained

$$
P_{F}^{R \star}\left(\left\{\epsilon_{p}^{R}, \epsilon_{q}^{R}\right\} ; \Upsilon\right) \geqslant P_{F, L B, 2}^{R \star}=h\left(F\left(\rho_{p}, \rho_{q}\right) ; \epsilon_{p}^{R}, \epsilon_{q}^{R}\right) .
$$

We have $P_{F, L B, 1}^{R \star} \geqslant P_{F, L B, 2}^{R \star}$, equal when $p=q=\frac{1}{2}$.

The above lemma also allows lower bounds for $P_{F}^{U \star}$. Because the bounds are obtained from the purification, Eq. (6) holds. Therefore, one can numerically obtain lower bounds by keeping track of the rescaling of the constraints. We will apply this lower bound on two ensembles of mixed states that are relevant for our later analyses of the channel case. To narrow down the ultimate performance, we also provide upper bounds on the minimum inconclusive probability by designing explicit measurement strategies.

Binary mixed states with depolarizing noise. We consider the approximate UD between an ensemble of two-qubit mixed states of equal priors,

$$
\rho_{ \pm}=\eta \psi_{ \pm}+\frac{1-\eta}{4} I_{4}
$$

where the pure states $\left|\psi_{ \pm}\right\rangle=(|00\rangle \pm|11\rangle) / \sqrt{2}$ are orthogonal maximal entangled states. This example of two-qubit states will also be utilized in the extension to channels in Sec. III B. The fidelity between states $\rho_{ \pm}$can be calculated analytically as

$$
F\left(\rho_{+}, \rho_{-}\right)=\frac{1}{2}\left(1-\eta+\sqrt{1+2 \eta-3 \eta^{2}}\right),
$$

and then the lower bound (15) can be analytically obtained for any error probability constraints. As shown in Fig. 2, the lower bound (red dashed line) is below unity at the exact UD limit of zero error and goes to zero earlier than the Helstrom limit point (red star). This is because the bound is in general nontight.

To provide an upper bound, we obtain the optimum strategy among a restricted class of strategies $\boldsymbol{\Pi}_{a, \theta}$ parametrized by $a, \theta$. Noticing the structure of the states, we design a measurement with POVMs $\Pi_{Q}=\left|\psi_{+}\right\rangle\left\langle\psi_{+}|+| \psi_{-}\right\rangle\left\langle\psi_{-}\right|$and $\Pi_{P}=I_{4}-\Pi_{Q}$ to separate them. If the result is $\Pi_{P}$, with probability $a \in[0,1]$ we decide inconclusive, with probability $1-a$ we perform random guess according to the prior. If the result is $\Pi_{Q}$, similar to Ref. [37] we consider the following POVMs parametrized by $\theta \in[\pi / 2, \pi]$ as the second step:

$$
\begin{gathered}
\Pi_{0}=\left(1-\frac{1}{\tan ^{2}(\theta / 2)}\right)|00\rangle\langle 00|, \\
\Pi_{ \pm}=\frac{1}{2 \sin ^{2}(\theta / 2)}\left|\phi_{ \pm \theta}\right\rangle\left\langle\phi_{ \pm \theta}\right|,
\end{gathered}
$$



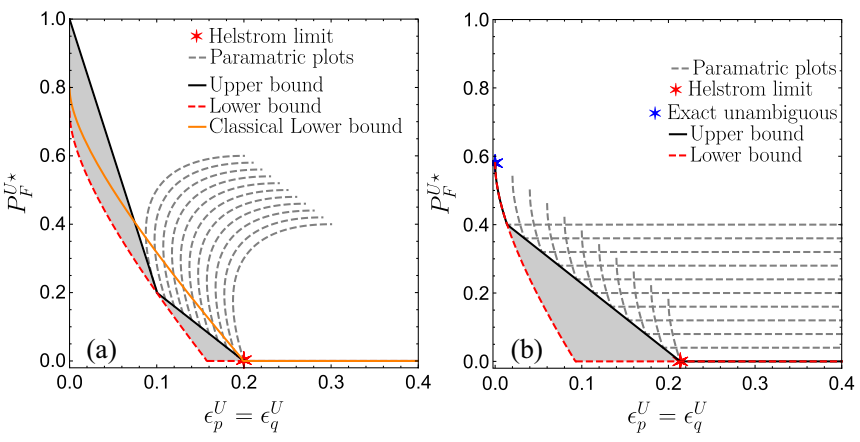

FIG. 2. Inconclusive probability $P_{F}^{U \star}$ for UD between binary mixed states, with equal prior $p=q=\frac{1}{2}$ and symmetric error probability constraint $\epsilon_{p}^{U}=\epsilon_{q}^{U}$. The actual value is in the gray region between the lower bound [red solid line, Eq. (15)] and the upper bound (black solid). The Helstrom limit is highlighted with the red star. We choose the parameters $\eta=0.6$ and $\langle p \mid q\rangle=0.3$ in both cases. (a) Mixed states with depolarizing noise. The upper bound is obtained from the convex hull of $(0,1)$, and the minimum over a family of strategies parametrized by $a$. The classical lower bound is given in Eq. (47). (b) Mixed states with a single common component. The upper bound and lower bound overlap when the error tolerance is small. The exact UD with inconclusive probability $\eta\langle p \mid q\rangle+(1-\eta)$ is marked in blue star. In both (a) and (b), the strategies can achieve the dashed gray curves for different $a=0,0.1, \ldots, 0.9,1$ from left to right.

with $\left|\phi_{\theta}\right\rangle=\cos (\theta / 2)|00\rangle+\sin (\theta / 2)|11\rangle$. The overall inconclusive probability and error probability are

$$
\begin{gathered}
P_{F}=\frac{a(1-\eta)}{2}+\frac{1+\eta}{4}\left(1-\frac{1}{\tan ^{2}(\theta / 2)}\right) \\
P_{E}=\frac{(1-\eta)(1-a)}{4}+\frac{1}{4 \sin ^{2}(\theta / 2)}\left[\frac{1+\eta}{2}-\eta \sin (\theta)\right] .
\end{gathered}
$$

One can then obtain an upper bound of $P_{F}^{U^{\star}}$ through convex hull of the above strategy and $(\mathbf{0}, 1)$, as shown by the black solid line in Fig. 2(a). It is worthy to point out that the strategies above can also achieve the Hellstrom limit $P_{H}=$ $(1-\eta) / 2$ (shown by the red star).

Binary mixed states with erasure. Consider a noise model, where two pure states in $\boldsymbol{\Upsilon}_{p, q}$ are mixed with a common pure orthogonal state $\phi$, via

$$
\rho_{x}=\eta|x\rangle\langle x|+(1-\eta) \phi,
$$

for $x=p, q$. We can evaluate the lower bound in Lemma 2 from the fidelity

$$
F=\eta|\langle q \mid p\rangle|+(1-\eta)=\eta \xi+(1-\eta) .
$$

As shown by the red dashed line in Fig. 2(b), the lower bound achieves the exact UD for the zero-error case, however, becomes zero earlier than the Helstrom limit (red star), which can be evaluated numerically.

To obtain an upper bound, we design a two-step measurement. First, we perform the POVM $\{\phi, I-\phi\}$. If the outcome is $\phi$, we conclude inconclusive with probability $a \in[0,1]$, and randomly guess according to the prior with probability $1-a$; otherwise, we proceed with the optimum strategy for the binary pure states case. The inconclusive probability and error constraints are given by

$$
\begin{gathered}
P_{F}^{U}=\eta P_{F}^{U \star}\left(\left\{\epsilon_{p}^{U \prime}, \epsilon_{q}^{U \prime}\right\} ; \Upsilon_{p, q}\right)+(1-\eta) a, \\
\epsilon_{p}^{U}=(1-\eta)(1-a) q+\eta \epsilon_{p}^{U \prime}, \\
\epsilon_{q}^{U}=(1-\eta)(1-a) p+\eta \epsilon_{q}^{U \prime},
\end{gathered}
$$

where the function $P_{F}^{U \star}\left(\cdot ; \Upsilon_{p, q}\right)$ is given by Eq. (10). By evaluating the above for different values of $a$ and $\epsilon_{p}^{U \prime}, \epsilon_{q}^{U^{\prime}}$, we can obtain the upper bound $P_{F}^{U}$ as a function of the overall error probabilities $\epsilon_{p}^{U}, \epsilon_{q}^{U}$, as shown by the black solid line in Fig. 2(b).

\section{Continuity}

Continuity is an important property for a physical quantifier. Quantum states and channels are theory models of physical processes. As accurate as it can be, a theoretical description has unavoidable deviations from the reality. In this scenario, a continuous quantifier robust against imperfections is desired. While exact UD is theoretically well studied, however, in practice when an ensemble of states is affected by a tiny bit of depolarizing noise, some ambiguity in the discrimination is unavoidable. While the property of exact UD is not continuous in quantum states, the relaxation to an approximate UD scenario allows a continuity bound (see Appendix E for a proof).

Lemma 3. Continuity of approximate UD: Consider two sets of states $\rho=\left\{\rho_{n}\right\}_{n=1}^{m}$ and $\boldsymbol{\rho}^{\prime}=\left\{\rho_{n}^{\prime}\right\}_{n=1}^{m}$, with one-norm deviation $\left\|\rho_{n}-\rho_{n}^{\prime}\right\| \leqslant \delta_{n}, 1 \leqslant n \leqslant m$. Given identical prior $\boldsymbol{P}=\left\{P_{n}\right\}_{n=1}^{m}$, the minimum failure probability $P_{F}^{U \star}(\boldsymbol{\epsilon} ;\{\boldsymbol{\rho}, \boldsymbol{P}\})$ as a function of the unrescaled tolerance $\epsilon$ satisfies the continuity

$$
\begin{gathered}
P_{F}^{U \star}(\boldsymbol{\epsilon} ;\{\boldsymbol{\rho}, \boldsymbol{P}\}) \in\left[P_{-}, P_{+}\right], \text {with } \\
P_{ \pm}=P_{F}^{U \star}\left(\boldsymbol{\epsilon} \mp \delta ;\left\{\boldsymbol{\rho}^{\prime}, \boldsymbol{P}\right\}\right) \pm \frac{1}{2} \boldsymbol{P} \cdot \boldsymbol{\delta},
\end{gathered}
$$

where we denote $\delta=\left\{\delta_{k}\right\}_{k=1}^{n}$.

Here, we focus on the unrescaled constraints. For rescaled constraints, the continuity lower bound is more complicated and thus we discuss it in Appendix E.

\section{APPROXIMATE UNAMBIGUOUS DISCRIMINATION BETWEEN CHANNELS}

Now, we proceed to address the approximate UD between quantum channels. In a channel discrimination scenario, one aims to perform hypothesis testing between a set of channels $\boldsymbol{E}=\left\{\mathcal{E}_{n}\right\}_{n=1}^{m}$, with prior probabilities $\boldsymbol{P}=\left\{p_{n}\right\}_{n=1}^{m}$. To do that, one inputs quantum states and measure the output. In general, one can adopt an entanglement-assisted adaptive protocol $\mathbb{P}_{u}$ of $u$ channel uses $[63,64]$, where each operation can depend on all previous measurement results at earlier times and unlimited entanglement can be utilized. In this protocol, one can access an unknown channel $\mathcal{E}$ for $u \geqslant 1$ times, as shown Fig. 3(a). With unlimited entanglement and unlimited ancillary systems allowed, all measurements can be pushed to the final output $\rho_{\mathcal{E}, u}$, by introducing controlled unitaries. In each round of probing, a subsystem $S_{k}, 1 \leqslant k \leqslant u$, in an arbitrary 


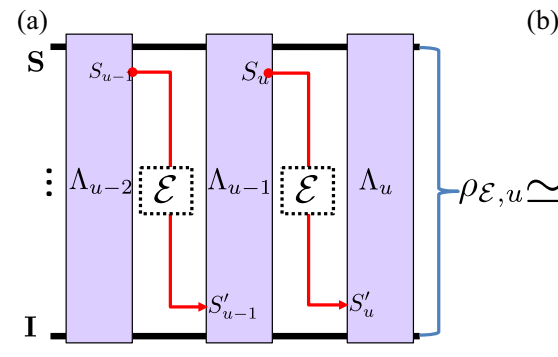

(b)

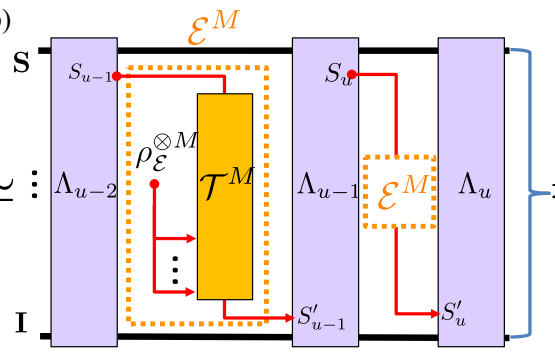

(c)

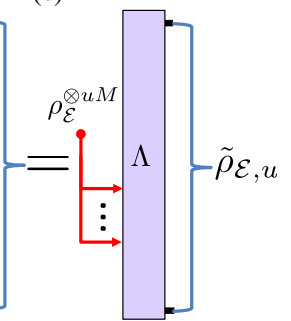

FIG. 3. Schematic of a $u$-step adaptive protocol $\mathbb{P}_{u}$ for channel discrimination.

quantum state, is sent through the channel $\mathcal{E}$ and the output $S_{k}^{\prime}$ is collected for later use. In an adaptive strategy, the quantum state of the probe $S_{k+1}$ in the next round can be produced by a unitary on all the previous collected outputs $\left\{S_{\ell}^{\prime}\right\}_{\ell=1}^{k}$ and an arbitrary number of ancilla in an arbitrary state.

The final decision of an adaptive protocol $\mathbb{P}_{u}$ is obtained from a measurement on the final outputs $\rho=\left\{\rho_{\mathcal{E}_{n}, u}\right\}_{n=1}^{m}$ (combining the prior $\boldsymbol{P}$ ); the problem of channel discrimination is reduced to a state discrimination problem. Therefore, for a fixed protocol $\mathbb{P}_{u}$, one can introduce the same set of performance metrics: the inconclusive probability $P_{F}$ in Eq. (1) and the error probability constraints in Eq. (3) or (4). However, the minimum inconclusive probability for $\epsilon$-approximate UD is now a constrained minimization over all sensing protocols,

$$
P_{F, u}^{X \star}(\boldsymbol{\epsilon} ;\{\boldsymbol{E}, \boldsymbol{P}\})=\min _{\mathbb{P}_{u}: \mathbb{E}_{X}} P_{F}\left(\boldsymbol{\epsilon} ;\{\boldsymbol{E}, \boldsymbol{P}\}, \mathbb{P}_{u}\right),
$$

for $X=U, R$ denoting the unrescaled or rescaled constraints, which is even more challenging than the state case in Eq. (5). Similar to the state case, the minimum error probability of adaptive channel MED [63,64] can also be given by $P_{u}(\{\boldsymbol{E}, \boldsymbol{P}\})=\min _{\boldsymbol{\epsilon}}^{\prime} \boldsymbol{P} \cdot \boldsymbol{\epsilon}$, where the minimization is under the constraint $P_{F, u}^{X \star}(\boldsymbol{\epsilon} ;\{\boldsymbol{E}, \boldsymbol{P}\})=0$.

In Sec. III A, we give a general lower bound to $P_{F, u}^{X \star}$, valid for any $u$-round adaptive sensing protocol. Then, we evaluate the lower bound for three examples in Sec. III B. We close this section by a discussion of the advantages from utilizing entanglement in the protocol.

\section{A. Lower bound on the ultimate limit of inconclusive probability}

To prove a lower bound of the inconclusive probability, we need to optimize over all possible protocols. To avoid such a challenging optimization, for each protocol $\mathbb{P}_{u}$, following Refs. [63,64] we can design another protocol to simulate its action. Suppose the protocol $\mathbb{P}_{u}$ on the $d$-dimensional channel $\mathcal{E}$ produces state $\rho_{\mathcal{E}, u}$, the simulation protocol produces an approximation $\tilde{\rho}_{\mathcal{E}, u}$ of $\rho_{\mathcal{E}, u}$, only relying on multiple copies of the Choi state

$$
\rho_{\mathcal{E}}=(\mathcal{E} \otimes \mathcal{I}) \zeta
$$

of the channel $\mathcal{E}$ rather than utilizing the original channel. Here, $\mathcal{I}$ is the identity channel and the pure state $|\zeta\rangle=$ $\sum_{\ell=0}^{d-1}|\ell, \ell\rangle / \sqrt{d}$ is a $d$-dimensional maximally entangled state. The protocol [see Figs. 3(b) and 3(c)] combines two techniques: channel simulation [66-69] and protocol stretching $[67,68]$.
First, we consider each channel use of $\mathcal{E}$ in the original sensing protocol. The action of channel $\mathcal{E}$ on the input can in general be approximated by a universal programmable quantum processor, given some program state that describes the channel $\mathcal{E}$ [69]. Note that here the quantum processor does not contain any information about the channel $\mathcal{E}$. In this paper, we consider processor as the general teleportation operation $\mathcal{T}^{M}$, as depicted in Fig. 3(b), while the program state is $M \geqslant 1$ copies of the Choi states $\rho_{\mathcal{E}}$. We denote such an approximation of the input-output relation as a channel $\mathcal{E}^{M}$.

In general, the teleportation operation $\mathcal{T}^{M}$ can be chosen as port-based teleportation (PBT) [70] and the number of Choi states $M$ can be optimized to obtain the best bound. In the special case of teleportation-covariant channels, where for each Pauli unitary $U$ and any quantum state $\rho$, one can find another unitary $V$ such that

$$
\mathcal{E}\left(U \rho U^{\dagger}\right)=V \mathcal{E}(\rho) V^{\dagger},
$$

one can simply use the direct teleportation to achieve an exact simulation with $m=1$ copy of Choi state.

The precision of such a channel simulation is quantified by the diamond norm deviation

$$
\Delta_{\mathcal{E}, M} \equiv\left\|\mathcal{E}-\mathcal{E}^{M}\right\|_{\diamond},
$$

where $\|A\|_{\diamond}=\sup _{\rho}\|A \otimes \mathcal{I}(\rho)\|$ is the diamond norm [51,71]. For teleportation-covariant channels, the simulation error $\Delta_{\mathcal{E}, M}=0$ for $M=1$; while for PBT simulation, the simulation error can in general be bounded by [63]

$$
\Delta_{\mathcal{E}, M} \leqslant \delta_{M, d} \equiv 2 d(d-1) M^{-1},
$$

which is valid for any number of ports $M \geqslant 1$ and any input dimension $d \geqslant 2$ for the channel. In general, other simulation protocols can also be used, and may potentially further decrease the simulation error and improve our overall lower bound.

By replacing the channel $\mathcal{E}$ with $\mathcal{E}^{M}$ in each step, one can obtain an overall protocol approximating the actual sensing protocol. Using the triangle inequality repeatedly, we can bound the deviation between the output state $\rho_{\mathcal{E}, u}$ of the actual protocol and the output state $\tilde{\rho}_{\mathcal{E}, u}$ of the simulated protocol as follows:

$$
\left\|\rho_{\mathcal{E}, u}-\tilde{\rho}_{\mathcal{E}, u}\right\| \leqslant u \Delta_{\mathcal{E}, M} .
$$

To provide a lower bound on the sensing performance, the final step is protocol stretching $[63,67,72]$. First, we notice that in each step of the protocol, the only channel-dependent component is the $M$ copies of the Choi states. Moreover, the overall $u M$ copies of the Choi states consumed in the $u$ 
steps can be directly prepared at the beginning of the entire protocol. Therefore, as depicted in Fig. 3(c), the simulated protocol can be organized into an equivalent protocol $\Lambda$ on the $u M$ copies of Choi states, so that the approximate output state $\tilde{\rho}_{\mathcal{E}, u}$ can be obtained as $\tilde{\rho}_{\mathcal{E}, u}=\Lambda\left(\rho_{\mathcal{E}}^{\otimes u M}\right)$. Combining this with Eq. (34) we have the overall error

$$
\left\|\rho_{\mathcal{E}, u}-\Lambda\left(\rho_{\mathcal{E}}^{\otimes u M}\right)\right\| \leqslant u \Delta_{\mathcal{E}, M} .
$$

With the above preparation, we can prove the following ultimate limit of approximate unambiguous channel discrimination.

Theorem 1. Consider arbitrary $m \geqslant 2 d$-dimensional quantum channels $\boldsymbol{E}=\left\{\mathcal{E}_{n}\right\}_{n=1}^{m}$ with prior probabilities $\boldsymbol{P}=\left\{p_{n}\right\}_{n=1}^{m}$. The failure probability of an arbitrary $u$-step adaptive protocol for $\boldsymbol{\epsilon}$-approximate UD satisfies

$$
\begin{aligned}
P_{F, u}^{U \star}(\boldsymbol{\epsilon} ;\{\boldsymbol{E}, \boldsymbol{P}\}) & \geqslant P_{F, u, L B, M}^{U}(\boldsymbol{\epsilon} ;\{\boldsymbol{E}, \boldsymbol{P}\}) \\
& \equiv P_{F}^{U \star}\left(\boldsymbol{\epsilon}+u \Delta_{\boldsymbol{E}, M} ;\left\{\rho_{\boldsymbol{E}}^{\otimes u M}, \boldsymbol{P}\right\}\right)-u \bar{\Delta}_{M} / 2,
\end{aligned}
$$

where we have used the notation $\Delta_{\boldsymbol{E}, M} \equiv\left\{u \Delta_{\mathcal{E}_{n}, M}\right\}_{n=1}^{m}$ and $\rho_{\boldsymbol{E}}^{\otimes u M} \equiv\left\{\rho_{\mathcal{E}_{n}}^{\otimes u M}\right\}_{n=1}^{m}$. Both the average simulation error $\bar{\Delta}_{M}=$ $\sum_{n} P_{n} \Delta_{\mathcal{E}_{n}, M}$ and each $\Delta_{\mathcal{E}_{n}, M}$ in $\Delta_{\boldsymbol{E}, M}$ can be replaced by the uniform error $\delta_{M, d}$ of Eq. (33).

In general, one optimizes $P_{F, u, L B, M}$ in Eq. (36) over $M$ to obtain the best lower bound. It is open whether this lower bound can be achievable in general. For the special case of a jointly teleportation-covariant ensemble of channels, where each channel $\mathcal{E}_{n} \in \boldsymbol{E}$ satisfies the relation in Eq. (31) with the same choice of $V$ for each $U$, the simulation becomes exact at $M=1$, therefore, we have the following corollary.

Corollary 1. Consider arbitrary $m \geqslant 2$ jointly teleportation-covariant channels $\boldsymbol{E}$ with prior probabilities $\boldsymbol{P}$. The minimum failure probability of an arbitrary $u$-step adaptive protocol for $\boldsymbol{\epsilon}$-approximate UD equals the corresponding formula computed over their Choi matrices

$$
P_{F, u}^{U \star}(\boldsymbol{\epsilon} ;\{\boldsymbol{E}, \boldsymbol{P}\})=P_{F}^{U \star}\left(\boldsymbol{\epsilon} ;\left\{\rho_{\boldsymbol{E}}^{\otimes u}, \boldsymbol{P}\right\}\right) .
$$

This is achievable by a nonadaptive entanglement-based strategy where $u$ copies of a maximally entangled state $\zeta$ are sent through the extended channel $\mathcal{E}_{n} \otimes \mathcal{I}$.

This means that for jointly teleportation-covariant channels, adaptivity is not necessary. As a by-product of our lower bound, we can extend the results of Ref. [54] to include adaptive strategies for teleportation-covariant channels; the $\epsilon=0$ case of Eq. (37) corresponds to the exact UD case. In fact, because for teleportation-covariant channels the simulations are exact, therefore Corollary 1 can be extended to the rescaled case

$$
P_{F, u}^{R \star}(\boldsymbol{\epsilon} ;\{\boldsymbol{E}, \boldsymbol{P}\})=P_{F}^{R \star}\left(\boldsymbol{\epsilon} ;\left\{\rho_{\boldsymbol{E}}^{\otimes u}, \boldsymbol{P}\right\}\right) .
$$

Although Eq. (36) in general reduces the channel problem to discrimination between Choi states, solving the minimum inconclusive probability of approximate UD for general mixed states still requires challenging optimizations. Here, we make use of the lower bound from Eq. (14) of Lemma 2 and Eq. (6), and further derive the following lower bound for binary quantum channels.
Lemma 4. Consider a pair of nonidentical channels $\left\{\mathcal{E}_{p}, \mathcal{E}_{q}\right\}$ with prior $\{p, q\}$, the minimum failure probability as a function of the unrescaled tolerance $\left\{\epsilon_{p}^{U}, \epsilon_{q}^{U}\right\}$ satisfies

$$
P_{F, u}^{U \star} \geqslant P_{F, u, L B, M}^{U} \equiv g\left(F_{\mathcal{E}_{p}, \mathcal{E}_{q}}^{u M} ; \epsilon_{p}^{R}, \epsilon_{q}^{R}\right)-u \bar{\Delta}_{M} / 2,
$$

with $\left\{\epsilon_{p}^{R}, \epsilon_{q}^{R}\right\}$ obtained from solving $\left\{\epsilon_{p}^{U}+u \Delta_{\mathcal{E}_{p}, M}, \epsilon_{q}^{U}+\right.$ $\left.u \Delta_{\mathcal{E}_{q}, M}\right\}=\left[1-g\left(F_{\mathcal{E}_{p}, \mathcal{E}_{q}}^{u M} ; \epsilon_{p}^{R}, \epsilon_{q}^{R}\right)\right]\left\{\epsilon_{p}^{R}, \epsilon_{q}^{R}\right\}$.

Here we utilized $F\left(\rho_{\mathcal{E}_{p}}^{\otimes u M}, \rho_{\mathcal{E}_{q}}^{\otimes u M}\right)=F^{u M}\left(\rho_{\mathcal{E}_{p}}, \rho_{\mathcal{E}_{q}}\right) \equiv$ $F_{\mathcal{E}_{p}, \mathcal{E}_{q}}^{u M}$, where we introduced $F_{\mathcal{E}_{p}, \mathcal{E}_{q}}$ as the fidelity between the Choi matrices for the two channels. The function $g$ is defined by Eq. (8). Note that when $p=q=\frac{1}{2}$, we can switch function $g$ in Eq. (39) to the analytical function $h$ given by Eq. (9) to have an analytical bound.

Similar to Corollary 1, when the two channels are teleportation covariant, we can let $M=1, \Delta_{\mathcal{E}_{p}, M}=\Delta_{\mathcal{E}_{q}, M}=0$ and obtain a simplified bound

$$
P_{F, u}^{\star} \geqslant P_{F, u, L B}^{U}=g\left(F_{\mathcal{E}_{p}, \mathcal{E}_{q}}^{u} ; \epsilon_{p}^{R}, \epsilon_{p}^{R}\right)
$$

with $\left\{\epsilon_{p}^{R}, \epsilon_{q}^{R}\right\} \quad$ obtained from solving $\left\{\epsilon_{p}^{U}, \epsilon_{q}^{U}\right\}=$ $\left[1-g\left(F_{\mathcal{E}_{p}, \mathcal{E}_{q}}^{u} ; \epsilon_{p}^{R}, \epsilon_{q}^{R}\right)\right]\left\{\epsilon_{p}^{R}, \epsilon_{q}^{R}\right\}$.

\section{B. Examples}

Now we evaluate the lower bound (39) in Lemma 4 in three examples. We first consider jointly teleportation-covariant channels, including noisy Pauli gates and quantum erasure channels. For these cases, Lemma 1 can be directly utilized and upper bounds can also be obtained by designing measurement schemes on the Choi states. Then, we consider the general case of amplitude-damping channels, which are not teleportation covariant.

Noisy Pauli gates. Single-qubit gates are a fundamental building block in quantum computers. It is therefore important to certify the type of gates being built. Practical gates are noisy, which makes the discrimination between different gates challenging. As an example, we consider the discrimination between two single-qubit Pauli gates in presence of depolarizing noise

$$
\mathcal{E}_{k}(\rho)=\eta \sigma_{k} \rho \sigma_{k}+(1-\eta) \frac{I_{2}}{2}, \quad k=Z, I
$$

with $\sigma_{Z}$ being the Pauli operator and $\sigma_{I}=I_{2}$ being identity. As Pauli channels, $\mathcal{E}_{Z}$ and $\mathcal{E}_{I}$ are jointly teleportation covariant, with Choi states

$$
\rho_{\mathcal{E}_{k}}=\eta\left|\Phi_{k}\right\rangle\left\langle\Phi_{k}\right|+(1-\eta) \frac{I_{4}}{4},
$$

where $\left|\Phi_{I}\right\rangle=|+\rangle$ and $\left|\Phi_{Z}\right\rangle=|-\rangle$ are maximally entangled. We see that the Choi states in Eq. (42) are identical to the binary mixed states with depolarizing noise described by Eq. (16). From Corollary 1, the results in Sec. II B immediately provide upper and lower bounds for the $u=1$ case of channel discrimination, as shown in Fig. 2(a). From the fidelity in Eq. (17), we can also obtain the lower bound in Eq. (40) for different $u$, as shown in Fig. 4(a).

Quantum erasure channels. We consider two different quantum erasure channels. Upon input $\rho$, each channel $\mathcal{E}_{k}$ gives the output

$$
\mathcal{E}_{k}(\rho)=\eta\left|e_{k}\right\rangle\left\langle e_{k}\right|+(1-\eta) \rho,
$$



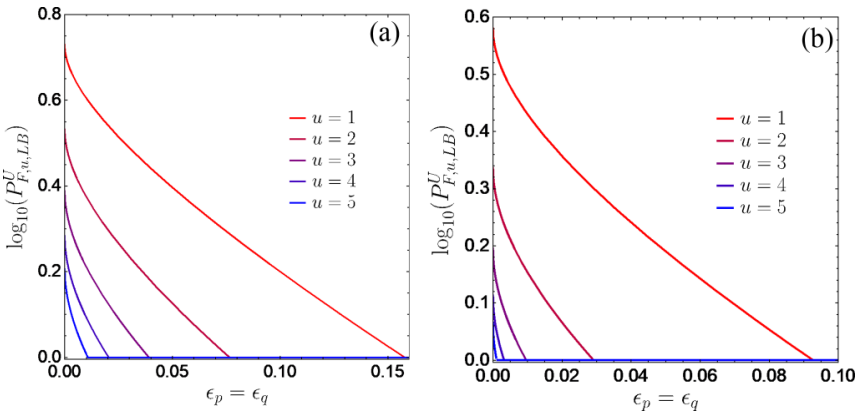

FIG. 4. Lower bound $P_{F, u, L B}^{U}$ vs the error tolerance for approximate UD between quantum channels of symmetric prior $p=q=\frac{1}{2}$. (a) Noisy Pauli gates with $\eta=0.6$, (b) quantum erasure channels with $\left\langle e_{1} \mid e_{2}\right\rangle=0.3$.

on the input $\rho$ for $k=1,2$, with two pure error states $\left\{\left|e_{1}\right\rangle,\left|e_{2}\right\rangle\right\}$ orthogonal to the input Hilbert space. Note that $\left\langle e_{1} \mid e_{2}\right\rangle$ can be nonzero in general. The two channels are jointly teleportation covariant, as the teleportation unitaries act only on the input Hilbert space. The Choi states

$$
\rho_{\mathcal{E}_{k}}=\eta\left|e_{k}\right\rangle\left\langle\left. e_{k}\right|_{E} \otimes \frac{I_{2}}{2}+(1-\eta) \mid \zeta\right\rangle\left\langle\left.\zeta\right|_{S I}\right.
$$

differ only by the error part, similar to the binary mixed state with a single common component considered in Eq. (22). Therefore, from Corollary 1, the upper bounds and lower bounds in Sec. II B can be utilized for the $u=1$ case here, which are calculated in Fig. 2(b). From the fidelity in Eq. (23), we can also obtain the lower bound in Eq. (40) for different $u$, as shown in Fig. 4(b).

Amplitude-damping channels. A quantum amplitude damping channel $\mathcal{A}_{r}$ with damping probability $r$ has the Kraus representation $\mathcal{A}_{r}(\rho)=\sum_{i=0,1} K_{i} \rho K_{i}^{\dagger}$, with operators $K_{0}=|0\rangle\langle 0|+\sqrt{1-r}| 1\rangle\langle 1|$ and $K_{1}=\sqrt{r}|0\rangle\langle 1|$. It is not telecovariant and its PBT simulation has nonzero error $\Delta_{\mathcal{A}_{r}, M}=$ $\xi_{M}[(1-r) / 2+\sqrt{1-r}]$, where $\xi_{M}$ is the constant given in Ref. [[63], Eq. (11)]. We consider the $u$-round approximate UD between $\mathcal{A}_{r_{q}}$ and $\mathcal{A}_{r_{p}}$. The fidelity between the Choi states of the channels can be obtained analytically $[63,64]$

$$
F_{\mathcal{A}_{r_{q}}, \mathcal{A}_{r_{p}}} \equiv\left[1+\sqrt{\left(1-r_{q}\right)\left(1-r_{p}\right)}+\sqrt{r_{q} r_{p}}\right] / 2 .
$$

We can therefore evaluate the lower bound $P_{F, u, L B, M}^{U}$ in Eq. (39) of Lemma 4. First, we optimize over $M$ and calculate the optimum lower bound $\max _{M} P_{F, u, L B, M}^{U}$ for different error constraints and number of rounds $u$ in Fig. 5. We see that as the number of rounds $u$ increases, the lower bound is decreasing as expected. To further understand the trend, we also evaluate the change of the lower bound $P_{F, u, L B, M}^{U}$ with $u$ for different fixed $M$ in Fig. 6 . The envelope of the lower bounds for all $M$ is the optimum lower bound we considered in Fig. 5. We see that when $M$ is small (red lines), the simulation error is large and causes the lower bound to be small, while when $M$ is large (blue lines), the abundance of Choi states makes the lower bound again small. For each $u$, one has an optimum $M$ to balance between the above two effects and provide the tightest lower bound.
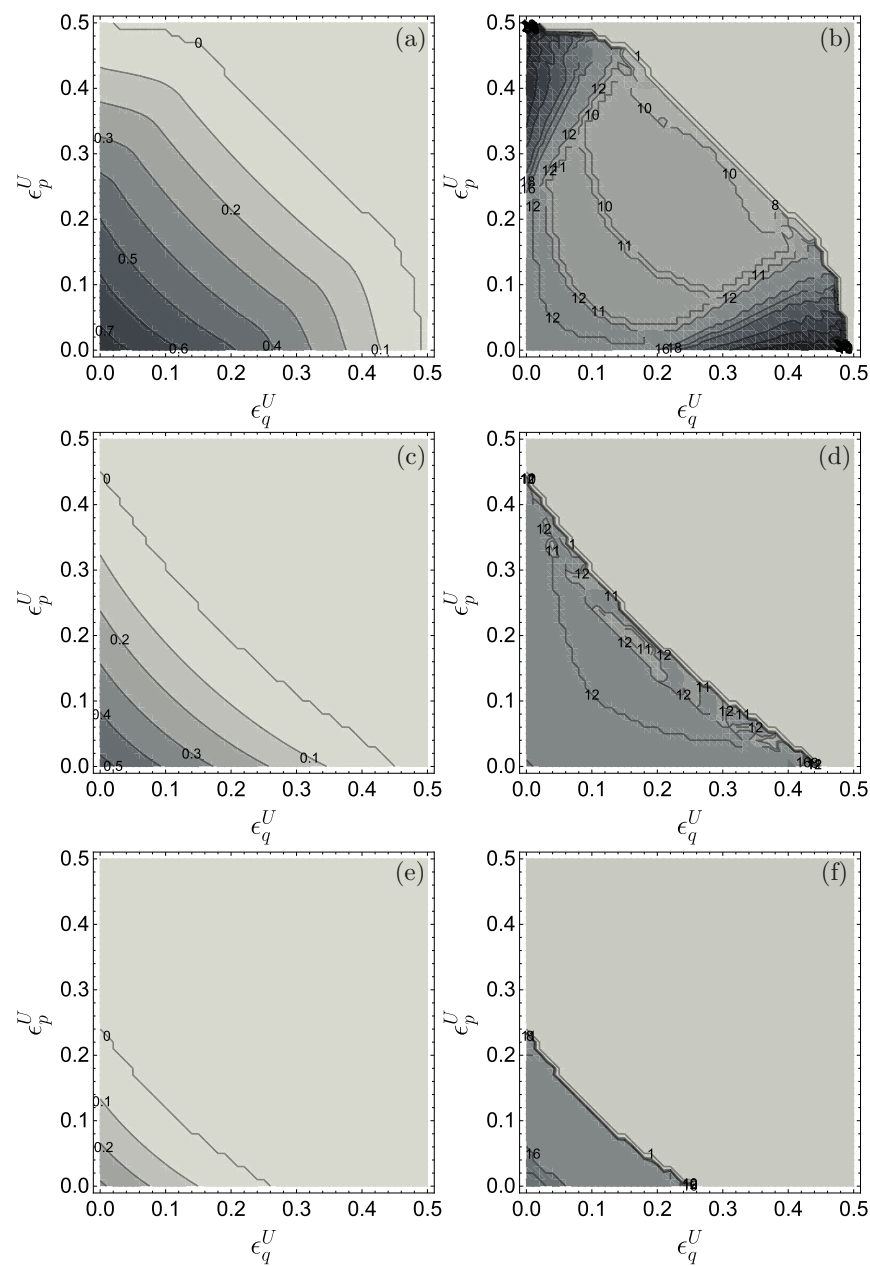

FIG. 5. Optimum lower bound $\max _{M} P_{F, u, L B, M}^{U}$ [Eq. (39) of Lemma 4] for a pair of quantum amplitude-damping channels with damping parameters $r_{q}=0.8, r_{p}=0.9$. (a), (b) $u=1$, (c), (d) $u=$ 2, (e), (f) $u=3$. We numerically scan over $\epsilon_{p}^{R}, \epsilon_{q}^{R}$ to obtain both $\epsilon_{p}^{U}, \epsilon_{q}^{U}$ and $P_{F, u, L B, M}^{U}$; then we optimize over $M$ for each $\epsilon_{p}^{U}, \epsilon_{q}^{U}$. The optimum $M$ are given in (b), (d), and (f).

Entanglement advantages over classical schemes. To better understand the effects of entanglement, here we consider nonadaptive classical strategies without entangled ancilla. To show the advantage of entanglement, we need upper bounds on the inconclusive probability of the entangled strategy and lower bounds on the classical correspondence. While efficient calculable lower bounds are provided in Lemma 4, upper bounds on the performance are hard to obtain, even for the approximate UD between the Choi states. Only in the $u=1$ case, we have upper bounds available, as depicted in Fig. 2. Therefore, we focus on the $u=1$ case.

First, we consider the noisy Pauli gates specified in Eq. (41). From the symmetry of the channel, we consider the input $|+\rangle$ in the classical strategy. Then one reduces the problem to states

$$
\rho_{Z}=\eta|-\rangle\left\langle-\left|+\frac{(1-\eta)}{2} I_{2}, \quad \rho_{I}=\eta\right|+\right\rangle\langle+|+\frac{(1-\eta)}{2} I_{2} .
$$

The fidelity between the states $F\left(\rho_{Z}, \rho_{I}\right)=\sqrt{1-\eta^{2}}$. From Eq. (40), the lower bound of the inconclusive probability can 

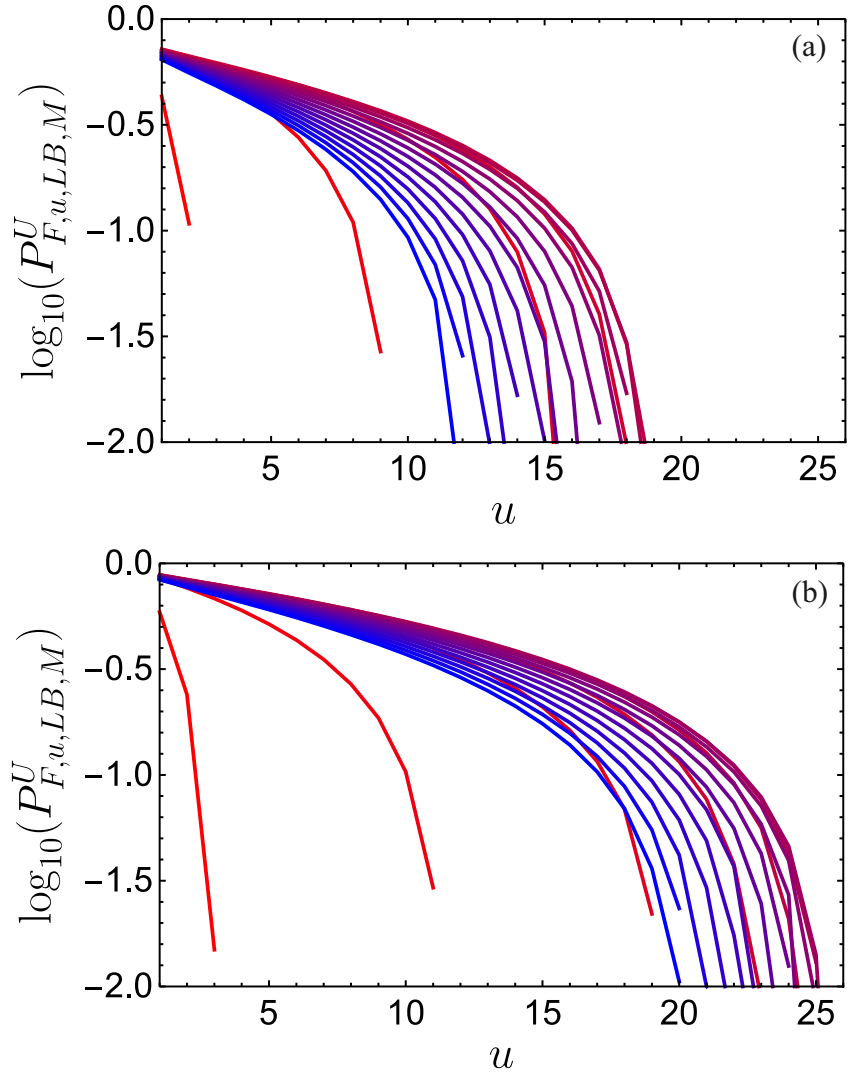

FIG. 6. Lower bound $P_{F, u, L B, M}^{U}$ in Eq. (39) of Lemma 4 vs the number of rounds $u . r_{q}=0.98, r_{p}=0.99$. (a) $\epsilon_{q}^{U}=0.1, \epsilon_{p}^{U}=0.1$ (b) $\epsilon_{q}^{U}=0.02, \epsilon_{p}^{U}=0.04$. Color indicates $M$, from red $(M=1)$ to blue $(M=81)$, in steps of $\Delta M=5$.

be obtained

$$
P_{F, u}^{\star} \geqslant P_{F, u, L B}^{U}=g\left(\sqrt{1-\eta^{2}} ; \epsilon_{p}^{R}, \epsilon_{p}^{R}\right) .
$$

We compare the above classical lower bound with the upper bound of the entangled strategies in Fig. 2. In the range around $0.07 \lesssim \epsilon_{p}^{U}=\epsilon_{q}^{U} \lesssim 0.2$, we can see an advantage from entanglement. We expect better entangled strategies to exist within the shaded region and a larger advantage is possible. The entanglement's benefit in presence of depolarizing noise resembles the advantage in quantum illumination [8]. Despite entanglement being fragile, its advantage over the performance achievable with only initial classical correlations survives noise.

Next, we consider the erasure channels specified in Eq. (44). From the symmetry of the channel, we can simply consider the input $|0\rangle$, leading to the output

$$
\rho_{k}=\eta\left|e_{k}\right\rangle\left\langle\left. e_{k}\right|_{E}+(1-\eta) \mid 0\right\rangle\langle 0|, \quad k=1,2 .
$$

The fidelity can be calculated as $F=\eta\left|\left\langle e_{1} \mid e_{2}\right\rangle\right|+(1-\eta)$, which is identical to the case with entanglement assistance. Therefore, the lower bound of the classical strategy coincides with the ultimate lower bound. And we are not able to show any entanglement advantage in this case, similar to the MED case in Ref. [64].

\section{CONCLUSIONS}

In this paper, we formulated the approximate unambiguous discrimination scenario for quantum states and quantum channels. For the binary pure states case, we are able to solve the minimum inconclusive probability as a function of the conclusive error probability constraints for an arbitrary prior. The minimum inconclusive probability satisfies convexity, continuity, and data processing, which makes it more friendly for both theory analyses and experimental realizations. For the channel case, we are able to prove an ultimate lower bound of the minimum inconclusive probability for any adaptive sensing protocols, based on the approximate unambiguous discrimination between the Choi states. For jointly teleportation-covariant channels, the lower bound can be achieved with maximum entangled inputs and no adaptive strategy is required.

\section{ACKNOWLEDGMENTS}

Q.Z. acknowledges funding from Defense Advanced Research Projects Agency (DARPA) under Young Faculty Award (YFA) Grant No. N660012014029 and University of Arizona. Q.Z. thanks S. Pirandola and J. Fiurášek for discussions.

\section{APPENDIX A: BINARY PURE STATES}

In the first example of binary pure states discrimination, we are able to solve $P_{F}^{R \star}(\boldsymbol{\epsilon} ; \mathbf{\Upsilon})$ as a function of $\boldsymbol{\epsilon}$ for an arbitrary prior. The equal prior exact UD is sovled in Refs. [28,65]. Here, we consider a general prior $\boldsymbol{P}=\{p, q\}$ and solve the approximate UD. Following Ref. [28], we adopt a measurement protocol assisted by an ancilla qubit in a pure state $\left|s_{0}\right\rangle$. A general unitary is applied on the ancilla and input to transform

$$
\begin{gathered}
\left|p s_{0}\right\rangle \rightarrow \alpha\left|p_{1} s_{1}\right\rangle+\beta\left|p_{2} s_{2}\right\rangle, \\
\left|q s_{0}\right\rangle \rightarrow \gamma\left|q_{1} s_{1}\right\rangle+\delta\left|q_{2} s_{2}\right\rangle,
\end{gathered}
$$

such that the output states of the ancilla is orthogonal, i.e., $\left\langle s_{1} \mid s_{2}\right\rangle=0$. Then, by measuring the ancilla in the $\left\{\left|s_{1}\right\rangle,\left|s_{2}\right\rangle\right\}$ bases, we can determine inconclusive when the outcome is $s_{2}$ and continue to perform a second measurement on the input when the outcome is $s_{1}$.

While Ref. [28] considers $\left\langle p_{1} \mid q_{1}\right\rangle=0$ to enable exact UD, we let $\left\langle p_{1} \mid q_{1}\right\rangle \geqslant 0$ in general. We consider a projective measurement along $\left|\phi_{\theta}\right\rangle=\cos \theta\left|p_{1}\right\rangle+\sin \theta\left|p_{1}^{\perp}\right\rangle$ and $\left|\phi_{\theta}^{\perp}\right\rangle=$ $\sin \theta\left|p_{1}\right\rangle-\cos \theta\left|p_{1}^{\perp}\right\rangle$ to trade off the error probabilities such that

$$
\begin{gathered}
P_{E \mid p}=\left|\left\langle p_{1} \mid \phi_{\theta}^{\perp}\right\rangle\right|^{2}=\sin ^{2} \theta \leqslant \epsilon_{p}, \\
P_{E \mid q}=\left|\left\langle q_{1} \mid \phi_{\theta}\right\rangle\right|^{2}=\left|\cos \theta\left\langle q_{1} \mid p_{1}\right\rangle+\sin \theta\left\langle q_{1} \mid p_{1}^{\perp}\right\rangle\right|^{2} \leqslant \epsilon_{q} .
\end{gathered}
$$

We can parametrize $\left\langle q_{1} \mid p_{1}^{\perp}\right\rangle=\sqrt{1-\left|\left\langle q_{1} \mid p_{1}\right\rangle\right|^{2}} e^{i \alpha}$. The first constraint is that there is a valid solution for $\theta, \alpha$, which leads to the constraint

$$
\left|\left\langle p_{1} \mid q_{1}\right\rangle\right| \in\left[\epsilon_{p q-}, \epsilon_{p q+}\right]
$$


where

$$
\epsilon_{p q \pm}=\left|\sqrt{\epsilon_{p}\left(1-\epsilon_{q}\right)} \pm \sqrt{\epsilon_{q}\left(1-\epsilon_{p}\right)}\right| .
$$

The other constraint is unitarity, which preserves the inner product

$$
\langle p \mid q\rangle=\alpha^{\star} \gamma\left\langle p_{1} \mid q_{1}\right\rangle+\delta^{\star} \beta\left\langle q_{2} \mid p_{2}\right\rangle .
$$

Because of norm preservation, $|\alpha|^{2}+|\beta|^{2}=|\gamma|^{2}+|\delta|^{2}=1$. Under these constraints, we want to minimize

$$
P_{F}=p|\beta|^{2}+q|\delta|^{2} .
$$

It is straightforward to see that to minimize $P_{F}$, we need $\left|\left\langle q_{2} \mid p_{2}\right\rangle\right|=1$ and the constraint in Eq. (A7) becomes

$$
|\langle p \mid q\rangle|=\sqrt{1-|\beta|^{2}} \sqrt{1-|\delta|^{2}}\left|\left\langle p_{1} \mid q_{1}\right\rangle\right|+|\delta||\beta| .
$$

We can reparametrize with angle parameters in $[0, \pi / 2]$ as $\beta=\sin \tilde{\beta}, \delta=\sin \tilde{\delta}$. Then we can obtain the optimization problem in Eq. (8). We can use inequality

$$
p \sin ^{2} \tilde{\beta}+q \sin ^{2} \tilde{\delta} \geqslant 2 \sqrt{p q} \sin \tilde{\beta} \sin \tilde{\delta},
$$

which is achievable when $p \sin ^{2} \tilde{\beta}=q \sin ^{2} \tilde{\delta}$. We can obtain a lower bound that leads to the solution in Eq. (9) via Lagrangian multiplier methods. Moreover, the only case that both Eqs. (A10) and (9) can be achieved is $p=q=\frac{1}{2}$, where we have

$$
P_{F}^{R \star}=1-\frac{1-|\langle p \mid q\rangle|}{1-\epsilon_{p q+}} .
$$

In fact, one can check that this result agrees with Ref. [34].

We can check both end points analytically. When $P_{F}=$ 0 , we have $\tilde{\alpha}=\tilde{\beta}=0$ and $\left|\left\langle p_{1} \mid q_{1}\right\rangle\right|=\langle p \mid q\rangle$, then simple optimization gives the Helstrom limit. In particular, for the equal-prior case, Eq. (7) leads to $P_{H}=\min \left(\epsilon_{p}^{R}+\epsilon_{q}^{R}\right) / 2$ under the constraint $|\langle p \mid q\rangle|=\epsilon_{p q+}$. From symmetry, it is achieved at $P_{H}=\epsilon_{p}=\epsilon_{q}=\left(1-\sqrt{1-4|\langle p \mid q\rangle|^{2}}\right) / 2$ which agrees with the Helstrom limit. When $\epsilon_{p}^{R}=\epsilon_{q}^{R}=0$, we can show that $\left\langle q_{1} \mid p_{1}\right\rangle=0$ and then the problem goes back to the exact UD case, and for the symmetric case one can obtain the exact UD results in Ref. [29].

To validate our numerical results of Eq. (8), in Fig. 7 we consider the equal prior case and compare the numerical results (black dots) and the analytical results of Eq. (A11) (orange dashed) along the line of $\epsilon_{p}=\epsilon_{q}$. A perfect agreement is found. Moreover, we analytically calculate the end points of exact UD (blue circle) and Hesltrom limit (red circle) and they also agree well with our results.

\section{APPENDIX B: PROOF OF EQ. (6)}

Let $P_{F}^{U \star}\left(\boldsymbol{\epsilon}^{U} ; \Upsilon \mathbf{\Upsilon}\right)=p_{f}$. Consider the rescaled constraints

$$
\boldsymbol{\epsilon}^{R}=\frac{1}{1-p_{f}} \boldsymbol{\epsilon}^{U} \text {. }
$$

First, because the same choice of measurement can achieve $p_{f}$ while satisfying the rescaled constraint of $\boldsymbol{\epsilon}^{R}$, we have

$$
P_{F}^{R \star}\left(\boldsymbol{\epsilon}^{R} ; \Upsilon\right) \leqslant p_{f} .
$$

Next, if

$$
P_{F}^{R \star}\left(\boldsymbol{\epsilon}^{R} ; \Upsilon\right)=p_{f}^{\prime}<p_{f},
$$

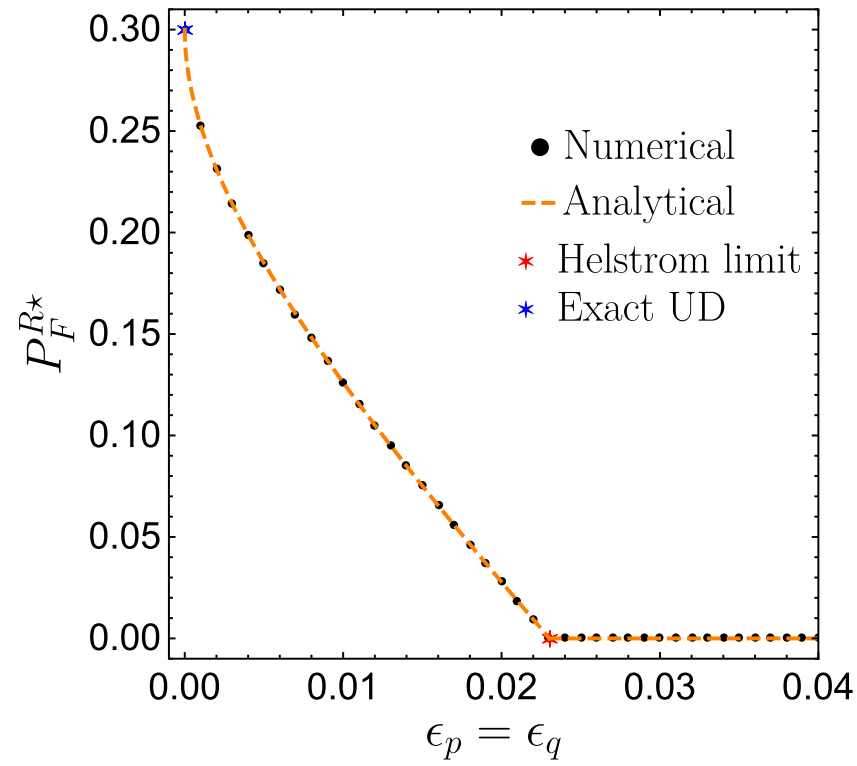

FIG. 7. $P_{F}^{R \star}$ : Comparison between numerical results and analytical results in the symmetric case of $p=q=\frac{1}{2}$ and $\langle p \mid q\rangle=0.3$.

then there is a corresponding

$$
\boldsymbol{\epsilon}^{U \prime}=\left(1-p_{f}^{\prime}\right) \boldsymbol{\epsilon}^{R}=\frac{1-p_{f}^{\prime}}{1-p_{f}} \boldsymbol{\epsilon}^{U}>\boldsymbol{\epsilon}^{U}
$$

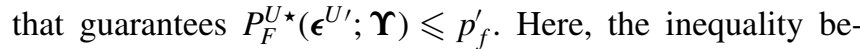
tween vectors means a set of item-wise inequalities. Figure 8 visualizes the setup by condensing all constraints into a single axis. The points $A=\left(\boldsymbol{\epsilon}^{U \prime}, p_{f}^{\prime}\right), B=\left(\boldsymbol{\epsilon}^{U}, p_{f}\right)$, and $C=(\mathbf{0}, 1)$ are on the same line. While point $B$ is on the curve $P_{F}^{U \star}$, the other two points $C$ and $A$ are upper bounds on $P_{F}^{U \star}$. From convexity in Lemma $1, C$ and $A$ have to be on the curve as well, namely,

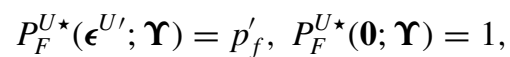

and the straight line $A C$ must be the optimum. If the $P_{F}^{U \star}(\boldsymbol{\epsilon} ; \Upsilon)$

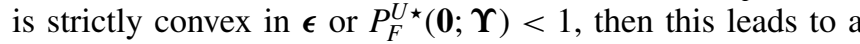

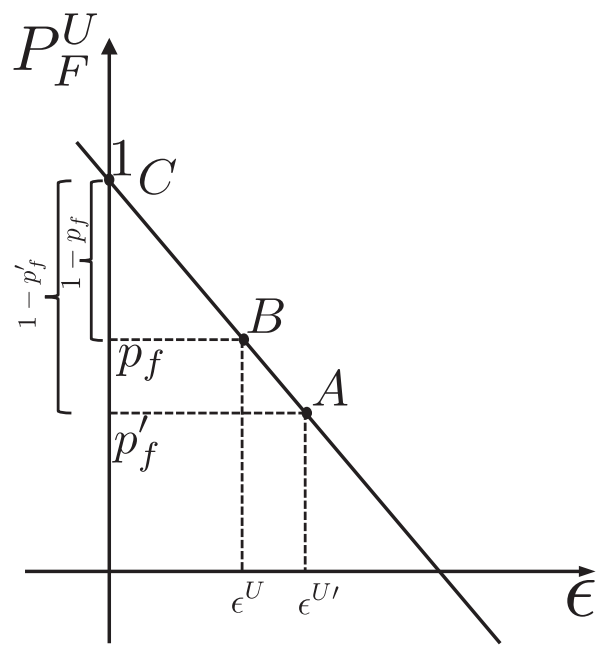

FIG. 8. Schematic of the proof of equivalence. 
contradiction, therefore, Eq. (B3) cannot be true and

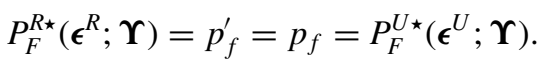

The only possible case would be a straight line $C A$ passing through $(0,1)$.

\section{APPENDIX C: PROOF OF LEMMA 1}

Suppose for each $\boldsymbol{\epsilon}^{(k)}$ the POVM achieving $P_{F}^{U \star}\left(\boldsymbol{\epsilon}^{(k)} ; \Upsilon \mathbf{\Upsilon}\right)$ is $\boldsymbol{\Pi}^{(k) \star}$. Then, we have

$$
P_{F}^{U \star}\left(\boldsymbol{\epsilon}^{(k)} ; \Upsilon \mathbf{\Upsilon}\right)=\sum P_{n} \operatorname{tr}\left(\Pi_{0}^{(k) \star} \rho_{n}\right)
$$

The constraints give

$$
P_{E \mid n}=1-\operatorname{tr}\left(\Pi_{0}^{(k) \star} \rho_{n}\right)-\operatorname{tr}\left(\Pi_{n}^{(k) \star} \rho_{n}\right) \leqslant \epsilon_{n}^{(k)} .
$$

We can define POVMs $\bar{\Pi}=\sum_{k} r_{k} \Pi^{(k) \star}$, which represents a strategy that performs the measurement represented by $\boldsymbol{\Pi}^{(k) \star}$ with probability $r_{k}$. It is easy to verify $\sum_{n} \bar{\Pi}_{n}=\sum_{k} r_{k} I=I$ and they are positive semidefinite. Then, the error probability

$$
\begin{aligned}
P_{E \mid n} & =1-\operatorname{tr}\left(\sum_{k} r_{k} \Pi_{0}^{(k) \star} \rho_{n}\right)-\operatorname{tr}\left(\sum_{k} r_{k} \Pi_{n}^{(k) \star} \rho_{n}\right) \\
& \leqslant \sum_{k} r_{k} \epsilon_{n}^{(k)} .
\end{aligned}
$$

The inconclusive probability

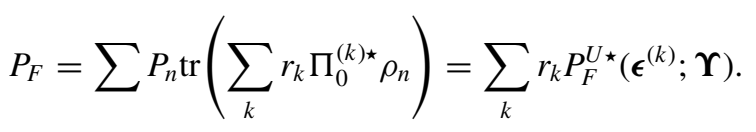

This strategy achieves the inconclusive probability $\sum_{k=1}^{K} r_{k} P_{F}^{U \star}\left(\boldsymbol{\epsilon}^{(k)} ; \mathbf{\Upsilon}\right)$ with error constraints $\sum_{k=1}^{K} r_{k} \boldsymbol{\epsilon}^{(k)}$; because better measurement can exist for the same error constraints, so we have Eq. (11) of Lemma 1.

\section{APPENDIX D: PROOF OF LEMMA 2}

From data processing we have

$$
\begin{aligned}
& P_{F}^{R \star}\left(\left\{\epsilon_{p}, \epsilon_{q}\right\} ;\left\{\left\{\rho_{p}, \rho_{q}\right\},\{p, q\}\right\}\right) \\
& \quad \geqslant P_{F}^{R \star}\left(\left\{\epsilon_{p}, \epsilon_{q}\right\} ;\left\{\left\{\psi_{\rho_{p}}, \psi_{\rho_{q}}\right\},\{p, q\}\right\}\right) \\
& \quad=g\left(\left|\left\langle\psi_{\rho_{p}} \mid \psi_{\rho_{q}}\right\rangle\right| ; \epsilon_{p}, \epsilon_{q}\right),
\end{aligned}
$$

where we used the binary pure states result in Eq. (8). Similar to Ref. [73], using Uhlmann's theorem that the maximum overlap between purification equals the fidelity $\left|\left\langle\psi_{\rho_{p}} \mid \psi_{\rho_{q}}\right\rangle\right| \leqslant$ $F\left(\rho_{p}, \rho_{q}\right) \equiv \operatorname{tr}\left(\sqrt{\sqrt{\rho_{q}} \rho_{p} \sqrt{\rho_{q}}}\right)$, also since $g$ is nondecreasing with $\left|\left\langle\psi_{\rho_{p}} \mid \psi_{\rho_{q}}\right\rangle\right|$ increasing, therefore one can obtain the tightest lower bound by taking

$$
\begin{aligned}
& P_{F}^{U \star}\left(\left\{\epsilon_{p}, \epsilon_{q}\right\} ;\left\{\left\{\rho_{p}, \rho_{q}\right\},\{p, q\}\right\}\right) \\
& \quad \geqslant P_{F, L B, 1}^{R \star}=g\left(F\left(\rho_{p}, \rho_{q}\right) ; \epsilon_{p}, \epsilon_{q}\right) .
\end{aligned}
$$

Then, further using the lower bound (9) for the binary pure states case, we can have the further lower bound $P_{F, L B, 2}^{R \star}$.

\section{APPENDIX E: PROOF OF LEMMA 3}

Here we extend the lemma to both rescaled and un-rescaled cases and then prove both results.
Lemma 5. Continuity of approximate UD: Consider two sets of states $\rho=\left\{\rho_{n}\right\}_{n=1}^{m}$ and $\rho^{\prime}=\left\{\rho_{n}^{\prime}\right\}_{n=1}^{m}$, with relative deviation $\left\|\rho_{n}-\rho_{n}^{\prime}\right\| \leqslant \delta_{n}, 1 \leqslant n \leqslant m$. Given identical prior $\boldsymbol{P}=\left\{P_{n}\right\}_{n=1}^{m}$, the minimum failure probability $P_{F}^{X \star}\left(\boldsymbol{\epsilon}^{X} ;\{\boldsymbol{\rho}, \boldsymbol{P}\}\right)$ as a function of the tolerance $\epsilon^{X}$ satisfies the continuity

$$
P_{F}^{X \star}\left(\boldsymbol{\epsilon}^{X} ;\{\boldsymbol{\rho}, \boldsymbol{P}\}\right) \geqslant P_{F}^{X \star}\left(\boldsymbol{\epsilon}^{X \prime} ;\left\{\boldsymbol{\rho}^{\prime}, \boldsymbol{P}\right\}\right)-\frac{1}{2} \boldsymbol{P} \cdot \boldsymbol{\delta},
$$

where we denote $\delta=\left\{\delta_{k}\right\}_{k=1}^{n}$, and use the notation that $X=U$ or $X=R$ represents the two different cases. The parameter for the unrescaled case

$$
\epsilon^{U^{\prime}}=\epsilon^{U}+\delta,
$$

while for the rescaled case

$$
\boldsymbol{\epsilon}^{R \prime} \equiv \frac{\boldsymbol{\delta}+\boldsymbol{\epsilon}^{R}\left[1-P_{F}^{R \star}\left(\boldsymbol{\epsilon}^{R} ;\{\boldsymbol{\rho}, \boldsymbol{P}\}\right)\right]}{1-P_{F}^{R \star}\left(\boldsymbol{\epsilon}^{R} ;\{\boldsymbol{\rho}, \boldsymbol{P}\}\right)-\frac{1}{2} \boldsymbol{P} \cdot \boldsymbol{\delta}} .
$$

Here we see that the unrescaled case has a much simpler continuity bound; in fact, by interchanging the variables, one can also show an upper bound as

$$
P_{F}^{U \star}\left(\boldsymbol{\epsilon}-\boldsymbol{\delta} ;\left\{\boldsymbol{\rho}^{\prime}, \boldsymbol{P}\right\}\right)+\frac{1}{2} \boldsymbol{P} \cdot \boldsymbol{\delta} \geqslant P_{F}^{U \star}(\boldsymbol{\epsilon} ;\{\boldsymbol{\rho}, \boldsymbol{P}\}),
$$

while in the second formalism, an upper bound will be complicated to obtain. form

To prove the lemma, we will use one-norm's variational

$$
\|A\|=2 \sup _{0 \leqslant P \leqslant I} \operatorname{Tr}[P A],
$$

so that $\left|\operatorname{Tr}\left[\left(\rho_{k}^{\prime}-\rho_{k}\right) \Pi\right]\right| \leqslant\left\|\rho_{k}^{\prime}-\rho_{k}\right\| / 2 \leqslant \delta_{k} / 2$ for any POVM element $\Pi$.

Consider a measurement described by the POVM elements $\Pi=\left\{\Pi_{n}\right\}_{n=0}^{m}$; below we show that its performance on the two ensembles of states is close. First, the inconclusive probability

$$
P_{F}(\boldsymbol{\rho})-P_{F}\left(\boldsymbol{\rho}^{\prime}\right)=\sum_{k=1}^{n} P_{k} \operatorname{Tr}\left(\Pi_{0}\left(\rho_{k}-\rho_{k}^{\prime}\right)\right),
$$

and therefore we have

$$
\left|P_{F}(\boldsymbol{\rho})-P_{F}\left(\boldsymbol{\rho}^{\prime}\right)\right| \leqslant \frac{1}{2} \boldsymbol{P} \cdot \boldsymbol{\delta} .
$$

The conditional error probability

$$
P_{E \mid k}(\boldsymbol{\rho})-P_{E \mid k}\left(\boldsymbol{\rho}^{\prime}\right)=\operatorname{Tr}\left(\left(\rho_{k}^{\prime}-\rho_{k}\right) \Pi_{k}\right)+\operatorname{Tr}\left(\left(\rho_{k}^{\prime}-\rho_{k}\right) \Pi_{0}\right),
$$

and therefore we have

$$
\left|P_{E \mid k}(\rho)-P_{E \mid k}\left(\rho^{\prime}\right)\right| \leqslant \delta_{k} .
$$

Now we look at the optimum solutions. For the first case, suppose for $\boldsymbol{\rho}$, the measurement achieves $P_{F}^{U \star}(\boldsymbol{\epsilon} ;\{\boldsymbol{\rho}, \boldsymbol{P}\})$. This means that

$$
P_{F}(\boldsymbol{\rho})=P_{F}^{U \star}(\boldsymbol{\epsilon} ;\{\boldsymbol{\rho}, \boldsymbol{P}\}), P_{E \mid k}(\boldsymbol{\rho}) \leqslant \epsilon_{k} .
$$

The performance of the same measurement on the other ensemble

$$
\begin{gathered}
P_{F}\left(\boldsymbol{\rho}^{\prime}\right) \leqslant P_{F}^{U \star}(\boldsymbol{\epsilon} ;\{\boldsymbol{\rho}, \boldsymbol{P}\})+\frac{1}{2} \boldsymbol{P} \cdot \boldsymbol{\delta}, \\
P_{E \mid k}\left(\boldsymbol{\rho}^{\prime}\right) \leqslant \delta_{k}+P_{E \mid k}\left(\boldsymbol{\rho}^{\prime}\right) \leqslant \delta_{k}+\epsilon_{k} .
\end{gathered}
$$

So we have an operating point that suffices to show

$$
P_{F}^{U \star}\left(\boldsymbol{\epsilon}+\delta ;\left\{\boldsymbol{\rho}^{\prime}, \boldsymbol{P}\right\}\right) \leqslant P_{F}^{U \star}(\boldsymbol{\epsilon} ;\{\boldsymbol{\rho}, \boldsymbol{P}\})+\frac{1}{2} \boldsymbol{P} \cdot \boldsymbol{\delta}
$$


because the minimum can only be smaller than $P_{F}\left(\rho^{\prime}\right)$.

For the second case, suppose for $\rho$ the measurement achieves $p_{f} \equiv P_{F}^{R \star}\left(\boldsymbol{\epsilon}^{R} ;\{\boldsymbol{\rho}, \boldsymbol{P}\}\right)$. This means that

$$
P_{F}(\boldsymbol{\rho})=p_{f}, P_{E \mid k}(\boldsymbol{\rho}) \leqslant \epsilon_{k}^{R}\left(1-p_{f}\right),
$$

and we have its performance on the other ensemble

$$
\begin{gathered}
P_{F}\left(\boldsymbol{\rho}^{\prime}\right) \leqslant p_{f}+\frac{1}{2} \boldsymbol{P} \cdot \boldsymbol{\delta}, \\
P_{E \mid k}\left(\boldsymbol{\rho}^{\prime}\right) \leqslant \delta_{k}+P_{E \mid k}\left(\boldsymbol{\rho}^{\prime}\right) \leqslant \delta_{k}+\epsilon_{k}^{R}\left(1-p_{f}\right) .
\end{gathered}
$$

The corresponding constraint

$$
\frac{P_{E \mid k}\left(\boldsymbol{\rho}^{\prime}\right)}{1-P_{F}\left(\boldsymbol{\rho}^{\prime}\right)} \leqslant \frac{\delta_{k}+\epsilon_{k}^{R}\left(1-p_{f}\right)}{1-p_{f}-\frac{1}{2} \boldsymbol{P} \cdot \boldsymbol{\delta}} .
$$

Note that the above is only true for $p_{f}+\frac{1}{2} \boldsymbol{P} \cdot \boldsymbol{\delta}<1$, however, when $p_{f}+\frac{1}{2} \boldsymbol{P} \cdot \boldsymbol{\delta} \geqslant 1$, the bound is meaningless and, therefore, we do not worry about that case. Therefore, the overall performance satisfies

$$
\begin{gathered}
P_{F}\left(\boldsymbol{\rho}^{\prime}\right) \leqslant p_{f}+\frac{1}{2} \boldsymbol{P} \cdot \boldsymbol{\delta}, \\
\frac{P_{E \mid k}\left(\boldsymbol{\rho}^{\prime}\right)}{1-P_{F}(\boldsymbol{\rho})} \leqslant \epsilon_{k}^{R^{\prime}} \equiv \frac{\delta_{k}+\epsilon_{k}^{R}\left(1-p_{f}\right)}{1-p_{f}-\frac{1}{2} \boldsymbol{P} \cdot \boldsymbol{\delta}} .
\end{gathered}
$$

This operating point suffices to give an upper bound

$$
P_{F}^{R \star}\left(\boldsymbol{\epsilon}^{R \prime} ;\left\{\boldsymbol{\rho}^{\prime}, \boldsymbol{P}\right\}\right) \leqslant P_{F}^{R \star}\left(\boldsymbol{\epsilon}^{R} ;\{\boldsymbol{\rho}, \boldsymbol{P}\}\right)+\frac{1}{2} \boldsymbol{P} \cdot \boldsymbol{\delta} .
$$

\section{APPENDIX F: PROOF OF THEOREM 1}

Proof. In this proof, we will simplify the notation and omit the identical prior $\boldsymbol{P}$ in the ensemble. First, by continuity equation (28) in Lemma 3 and applying inequality (35) on each channel, we have

$$
\begin{gathered}
P_{F}^{U \star}\left(\boldsymbol{\epsilon} ;\left\{\rho_{\mathcal{E}_{n}, u}\right\}_{n=1}^{m}\right) \geqslant \\
P_{F}^{U \star}\left(\left\{\epsilon+u \Delta_{\mathcal{E}_{n}, M}\right\}_{n=1}^{m} ;\left\{\Lambda\left(\rho_{\mathcal{E}_{n}}^{\otimes u M}\right)\right\}_{n=1}^{m}\right) \\
-\frac{1}{2} \sum_{n=1}^{m} u P_{n} \Delta_{\mathcal{E}_{n}, M}, \\
\geqslant P_{F}^{U \star}\left(\left\{\epsilon+u \Delta_{\mathcal{E}_{n}, M}\right\}_{n=1}^{m} ;\left\{\rho_{\mathcal{E}_{n}}^{\otimes u M}\right\}_{n=1}^{m}\right)-\frac{u}{2} \sum_{n=1}^{m} P_{n} \Delta_{\mathcal{E}_{n}, M} .
\end{gathered}
$$

In the last step, we utilized the data-processing inequality (12).

The above inequality holds for any output states $\left\{\rho_{\mathcal{E}_{n}, u}\right\}_{n=1}^{m}$, and therefore holds for an arbitrary adaptive protocol $\mathbb{P}_{u}$.
[1] S. Pirandola, B. R. Bardhan, T. Gehring, C. Weedbrook, and S. Lloyd, Advances in photonic quantum sensing, Nat. Photonics 12, 724 (2018).

[2] I. Ruo Berchera and I. P. Degiovanni, Quantum imaging with sub-poissonian light: Challenges and perspectives in optical metrology, Metrologia 56, 024001 (2019).

[3] C. L. Degen, F. Reinhard, and P. Cappellaro, Quantum sensing, Rev. Mod. Phys. 89, 035002 (2017).

[4] V. Giovannetti, S. Lloyd, and L. Maccone, Advances in quantum metrology, Nat. Photonics 5, 222 (2011).

[5] V. Giovannetti, S. Lloyd, and L. Maccone, Quantum-enhanced positioning and clock synchronization, Nature (London) 412, 417 (2001).

[6] Q. Zhuang and S. Pirandola, Entanglement-enhanced testing of multiple quantum hypotheses, Commun. Phys. 3, 103 (2020).

[7] S. Lloyd, Enhanced sensitivity of photodetection via quantum illumination, Science 321, 1463 (2008).

[8] S.-H. Tan, B. I. Erkmen, V. Giovannetti, S. Guha, S. Lloyd, L. Maccone, S. Pirandola, and J. H. Shapiro, Quantum Illumination with Gaussian States, Phys. Rev. Lett. 101, 253601 (2008).

[9] S. Barzanjeh, S. Guha, C. Weedbrook, D. Vitali, J. H. Shapiro, and S. Pirandola, Microwave Quantum Illumination, Phys. Rev. Lett. 114, 080503 (2015).

[10] Z. Zhang, S. Mouradian, F. N. C. Wong, and J. H. Shapiro, Entanglement-Enhanced Sensing in a Lossy and Noisy Environment, Phys. Rev. Lett. 114, 110506 (2015).

[11] Q. Zhuang, Z. Zhang, and J. H. Shapiro, Optimum Mixed-State Discrimination for Noisy Entanglement-Enhanced Sensing, Phys. Rev. Lett. 118, 040801 (2017).

[12] S. Pirandola, Quantum Reading of a Classical Digital Memory, Phys. Rev. Lett. 106, 090504 (2011).

[13] Q. Zhuang, Z. Zhang, and J. H. Shapiro, Distributed quantum sensing using continuous-variable multipartite entanglement, Phys. Rev. A 97, 032329 (2018).
[14] T. J. Proctor, P. A. Knott, and J. A. Dunningham, Multiparameter Estimation in Networked Quantum Sensors, Phys. Rev. Lett. 120, 080501 (2018).

[15] W. Ge, K. Jacobs, Z. Eldredge, A. V. Gorshkov, and M. Foss-Feig, Distributed Quantum metrology with Linear Networks and Separable Inputs, Phys. Rev. Lett. 121, 043604 (2018).

[16] X. Guo, C. R. Breum, J. Borregaard, S. Izumi, M. V. Larsen, T. Gehring, M. Christandl, J. S. Neergaard-Nielsen, and U. L. Andersen, Distributed quantum sensing in a continuousvariable entangled network, Nat. Phys. 16, 281 (2020).

[17] Y. Xia, W. Li, W. Clark, D. Hart, Q. Zhuang, and Z. Zhang, Demonstration of a Reconfigurable Entangled Radio-Frequency Photonic Sensor Network, Phys. Rev. Lett. 124, 150502 (2020).

[18] Q. Zhuang, J. Preskill, and L. Jiang, Distributed quantum sensing enhanced by continuous-variable error correction, New J. Phys. 22, 022001 (2020).

[19] H. Shi, Z. Zhang, S. Pirandola, and Q. Zhuang, EntanglementAssisted Absorption Spectroscopy, Phys. Rev. Lett. 125, 180502 (2020).

[20] LIGO Scientific Collaboration, Observation of Gravitational Waves From a Binary Black Hole Merger, Phys. Rev. Lett. 116, 061102 (2016).

[21] L. S. Collaboration, A gravitational wave observatory operating beyond the quantum shot-noise limit, Nat. Phys. 7, 962 (2011).

[22] L. S. Collaboration, Quantum-Enhanced Advanced Ligo Detectors in the Era of Gravitational-Wave Astronomy, Phys. Rev. Lett. 123, 231107 (2019).

[23] C. Helstrom, Minimum mean-squared error of estimates in quantum statistics, Phys. Lett. A 25, 101 (1967).

[24] C. Helstrom, Quantum Detection and Estimation Theory, Mathematics in Science and Engineering: A Series of Monographs and Textbooks (Academic, New York, 1976). 
[25] H. Yuen and M. Lax, Multiple-parameter quantum estimation and measurement of nonselfadjoint observables, IEEE Trans. Inf. Theory 19, 740 (1973).

[26] A. Holevo, Probabilistic and Statistical Aspects of Quantum Mechanics (North-Holland, Amsterdam, 1982).

[27] I. D. Ivanovic, How to differentiate between non-orthogonal states, Phys. Lett. A 123, 257 (1987).

[28] D. Dieks, Overlap and distinguishability of quantum states, Phys. Lett. A 126, 303 (1988).

[29] A. Peres, How to differentiate between non-orthogonal states, Phys. Lett. A 128, 19 (1988).

[30] B. Huttner, A. Muller, J. D. Gautier, H. Zbinden, and N. Gisin, Unambiguous quantum measurement of nonorthogonal states, Phys. Rev. A 54, 3783 (1996).

[31] A. Chefles, Unambiguous discrimination between linearly independent quantum states, Phys. Lett. A 239, 339 (1998).

[32] M. Dušek, M. Jahma, and N. Lütkenhaus, Unambiguous state discrimination in quantum cryptography with weak coherent states, Phys. Rev. A 62, 022306 (2000).

[33] L.-M. Duan and G.-C. Guo, Probabilistic cloning and identification of linearly independent quantum states, Phys. Rev. Lett. 80, 4999 (1998).

[34] A. Chefles and S. M. Barnett, Strategies for discriminating between non-orthogonal quantum states, J. Mod. Opt. 45, 1295 (1998).

[35] C.-W. Zhang, C.-F. Li, and G.-C. Guo, General strategies for discrimination of quantum states, Phys. Lett. A 261, 25 (1999).

[36] Y. C. Eldar, Mixed-quantum-state detection with inconclusive results, Phys. Rev. A 67, 042309 (2003).

[37] J. Fiurášek and M. Ježek, Optimal discrimination of mixed quantum states involving inconclusive results, Phys. Rev. A 67, 012321 (2003).

[38] K. Nakahira, K. Kato, and T. S. Usuda, Generalized quantum state discrimination problems, Phys. Rev. A 91, 052304 (2015).

[39] M. A. P. Touzel, R. B. A. Adamson, and A. M. Steinberg, Optimal bounded-error strategies for projective measurements in nonorthogonal-state discrimination, Phys. Rev. A 76, 062314 (2007).

[40] K. Nakahira, T. S. Usuda, and K. Kato, Finding optimal solutions for generalized quantum state discrimination problems, IEEE Trans. Inf. Theory 63, 7845 (2016).

[41] S. Croke, E. Andersson, S. M. Barnett, C. R. Gilson, and J. Jeffers, Maximum Confidence Quantum Measurements, Phys. Rev. Lett. 96, 070401 (2006).

[42] U. Herzog, Discrimination of two mixed quantum states with maximum confidence and minimum probability of inconclusive results, Phys. Rev. A 79, 032323 (2009).

[43] A. Hayashi, T. Hashimoto, and M. Horibe, State discrimination with error margin and its locality, Phys. Rev. A 78, 012333 (2008).

[44] K. Nakahira, T. S. Usuda, and K. Kato, Finding optimal measurements with inconclusive results using the problem of minimum error discrimination, Phys. Rev. A 91, 022331 (2015).

[45] J. Combes and C. Ferrie, Cost of postselection in decision theory, Phys. Rev. A 92, 022117 (2015).

[46] J. Fiurášek, Optimal probabilistic estimation of quantum states, New J. Phys. 8, 192 (2006).
[47] B. Gendra, E. Ronco-Bonvehi, J. Calsamiglia, R. Muñoz-Tapia, and E. Bagan, Quantum Metrology Assisted by Abstention, Phys. Rev. Lett. 110, 100501 (2013).

[48] B. Gendra, E. Ronco-Bonvehi, J. Calsamiglia, R. Muñoz-Tapia, and E. Bagan, Optimal parameter estimation with a fixed rate of abstention, Phys. Rev. A 88, 012128 (2013).

[49] O. Hirota, Properties of quantum communication with received quantum state control, Opt. Commun. 67, 204 (1988).

[50] A. Chefles, Quantum state discrimination, Contemp. Phys. 41, 401 (2000).

[51] A. Y. Kitaev, Quantum computations: Algorithms and error correction, Russ. Math. Surv. 52, 1191 (1997).

[52] A. Acín, E. Jané, and G. Vidal, Optimal estimation of quantum dynamics, Phys. Rev. A 64, 050302(R) (2001).

[53] M. F. Sacchi, Entanglement can enhance the distinguishability of entanglement-breaking channels, Phys. Rev. A 72, 014305 (2005).

[54] G. Wang and M. Ying, Unambiguous discrimination among quantum operations, Phys. Rev. A 73, 042301 (2006).

[55] M. Hayashi, Quantum Information Theory: Mathematical Foundation (Springer, Berlin, 2016).

[56] Y. Feng, R. Duan, and M. Ying, Unambiguous discrimination between mixed quantum states, Phys. Rev. A 70, 012308 (2004).

[57] A. Chefles and S. M. Barnett, Optimum unambiguous discrimination between linearly independent symmetric states, Phys. Lett. A 250, 223 (1998).

[58] U. Herzog, Optimum unambiguous discrimination of two mixed states and application to a class of similar states, Phys. Rev. A 75, 052309 (2007).

[59] S. Pang and S. Wu, Optimum unambiguous discrimination of linearly independent pure states, Phys. Rev. A 80, 052320 (2009).

[60] M. Kleinmann, H. Kampermann, and D. Bruß, Structural approach to unambiguous discrimination of two mixed quantum states, J. Math. Phys. 51, 032201 (2010).

[61] J. A. Bergou, U. Futschik, and E. Feldman, Optimal Unambiguous Discrimination of Pure Quantum States, Phys. Rev. Lett. 108, 250502 (2012).

[62] S. Bandyopadhyay, Unambiguous discrimination of linearly independent pure quantum states: Optimal average probability of success, Phys. Rev. A 90, 030301(R) (2014).

[63] S. Pirandola, R. Laurenza, C. Lupo, and J. L. Pereira, Fundamental limits to quantum channel discrimination, npj Quantum Inf. 5, 50 (2019).

[64] Q. Zhuang and S. Pirandola, Ultimate limits for multiple quantum channel discrimination, Phys. Rev. Lett. 125, 080505 (2020)

[65] G. Jaeger and A. Shimony, Optimal distinction between two non-orthogonal quantum states, Phys. Lett. A 197, 83 (1995).

[66] M. A. Nielsen and I. L. Chuang, Programmable Quantum Gate Arrays, Phys. Rev. Lett. 79, 321 (1997).

[67] S. Pirandola, R. Laurenza, C. Ottaviani, and L. Banchi, Fundamental limits of repeaterless quantum communications, Nat. Commun. 8, 15043 (2017).

[68] S. Pirandola, S. L. Braunstein, R. Laurenza, C. Ottaviani, T. P. W. Cope, G. Spedalieri, and L. Banchi, Programmable quantum gate arrays, Quantum Sci. Technol. 3, 035009 (2018). 
[69] L. Banchi, J. Pereira, S. Lloyd, and S. Pirandola, Convex optimization of programmable quantum computers, npj Quantum Inf. 6, 42 (2020).

[70] S. Ishizaka and T. Hiroshima, Asymptotic Teleportation Scheme as a Universal Programmable Quantum Processor, Phys. Rev. Lett. 101, 240501 (2008).
[71] V. I. Paulsen, Completely Bounded Maps and Operator Algebras (Cambridge University Press, Cambridge, 2002).

[72] S. Pirandola and C. Lupo, Ultimate Precision of Adaptive Noise Estimation, Phys. Rev. Lett. 118, 100502 (2017).

[73] T. Rudolph, R. W. Spekkens, and P. S. Turner, Unambiguous discrimination of mixed states, Phys. Rev. A 68, 010301(R) (2003). 